\title{
CRITICALITY REFERENCE BENCHM:RK CALCULATIONS FOR BURNUP CREDIT USING SPENT FUEL ISOTOPICS
}

\section{S. M. Bowman}

Computing and Telecommunications Division Nuclear Engineering Applications Department

Oak Ridge National Laboratory

Oak Ridge, Tennessee

April 1991

NOTICE: This document contains information of a preliminary nature. It is subject to revision or correction and therefore does not represent a final report.

Prepared under the direction of

Sandia National Laboratories

under subcontract 66-0162 with Oak Ridge National Laboratory

MARTIN MARIETTA ENERGY SYSTEMS, INC. managing the

Oak Ridge National Laboratory Oak Ridge Y-12 Plant

Oak Ridge Gaseous Diffusion Plant Paducah Gascous Diffusion Plant for the

U.S. DEPARTMENT OF ENERGY

under contract DE-AC05-84OR21400 


\section{INTRODUCTION}

To date, criticality analyses performed in support of the certification of spent fuel casks in the United States do not take credit for the reactivity reduction that results from burnup. By taking credit for the fuel burnup, commonly referred to as "burnup credit," the fuel loading capacity of these casks can be increased.

One of the difficulties in implementing burnup credit in criticality analyses is that there have been no critical experiments performed with spent fuel which can be used for computer code validation. In lieu of that, a reference problem set of fresh fuel critical experiments which model various conditions typical of light water reactor (LWR) transportation and storage casks has been identified and used in the validation of SCALE-4. ${ }^{1.2}$ This report documents the use of this same problem set to perform spent fuel criticality benchmark calculations by replacing the actual fresh fuel isotopics from the experiments with six different sets of calculated spent fuel isotopics. The SCALE-4 modules SAS2H ${ }^{3}$ and CSAS4 4 were used to perform the analyses. These calculations do not model actual critical experiments. The calculated $k$-effectives are not supposed to equal unity and will vary depending on the initial enrichment and burnup of the calculated spent fuel isotopics.

\section{GENERATION OF SPEN T FUEL ISOTOPICS}

LWR spent fuel isotopies were generated using the SAS2H module in SCALE-4. Three initial enrichments of 3.0,3.75, and 4.5 weight percent (wt \%) ${ }^{235} \mathrm{U}$ and two burnups of 18,000 and 25,000 MWd/MTU were selected to give a total of six differ:ent enrichment/burnup combinatiors.

The initial isotopic distribution assumed for each enrichment is presented in Table 1 . The $w 7 \%$ of ${ }^{25} U$ and ${ }^{26} U$ for the lowest enrichment $\left(3.0 \mathrm{wt} \%{ }^{235} U\right)$ were caiculated using the following formulae:

$$
\begin{aligned}
& w \%{ }^{25} U=0.007731\left(w t \%{ }^{35} U\right)^{108370} \\
& w \%{ }^{256} U=0.0046\left(w t \%{ }^{235} U\right)
\end{aligned}
$$

These formulac are empirical fits of measuied data for LWR fuel over a range of 2.56 to 3.13 wi \%. The values for the two higher enrichments ( 3.75 and $\left.4.5 \mathrm{wt} \%{ }^{235} \mathrm{U}\right)$ are based on extrapolation of measured data for 3.8 and $4.0 \mathrm{wn} \%{ }^{235} \mathrm{U}$ fucl. 
Table 1. Initial fuel isotopic distributions

\begin{tabular}{lccc}
\hline wt $\%{ }^{35} U$ & ut $\%{ }^{34} U$ & wt $\%{ }^{36} U$ & ut $\%{ }^{38} U$ \\
\hline 3.0 & 0.0254 & 0.0138 & 96.9608 \\
3.75 & 0.0294 & 0.0233 & 96.1973 \\
4.5 & 0.0358 & 0.0278 & 95.4364 \\
\hline
\end{tabular}

The fuel assembly model used in SAS2H was a standard Westinghouse $17 \times 17$ design. The data used to model the assembly are given in Table 2. The spent fuel isotopes tracked in SAS2H are listed in Table 3. The input xenon concentration was $6.72 \times 10^{-9}$ atoms/b-cm.

Table 2. Fuel assembl jata

\begin{tabular}{ll}
\hline Parameter & Data \\
\hline Assembly general data & Westinghouse \\
Designer & $17 \times 17$ \\
Lattice & 570 \\
Water temperature, K & 0.733 \\
Water density, g-cm.3 & 750 \\
Soluble boron, cycle av, ppm (wt) & 264 \\
Number of fuel rods & 24 \\
Number of guide tubes & 1 \\
Number of instrument tubes & $21.50364(8.466)$ \\
Assembly pitch cm (in.) & \\
& \\
Fuel rod data & UO \\
Type fuel pellet & 95 \\
Pellet stack density, \% TD & $1.25984(0.496)$ \\
Rod pitch, cm (in.) & $0.94966(0.374)$ \\
Rod OD, cm (in.) & $0.83566(0.329)$ \\
Rod ID, cm (in.) & $0.81915(0.3225)$ \\
Pellet diameter, cm (in.) & $365.76(144)$ \\
Active fuel length, cm (in.) & 811 \\
Effective fuel temperature, K & 620 \\
Clad temperature, K & Zircalloy \\
Clad material & \\
& \\
Guide tube data & \\
Inner radius, cm (in.) & $0.5715(0.45)$ \\
Outer radius, cm (in.) & $0.61214(0.482)$ \\
Tube material & Zircalloy \\
\hline
\end{tabular}


Table 3. Spent fuel isotopes tracked in SAS2H

\begin{tabular}{|c|c|c|c|}
\hline $\mathrm{Kr}_{\mathbf{T}} \mathbf{8 5}$ & Ag-109 & Nd-143 & U-236* \\
\hline Sr -90 & Sb-124 & Nd-145 & U-238* \\
\hline Y-89 & Xe-131 & $\mathrm{Pm}-147$ & Np-237* \\
\hline Mo-95 & $\mathrm{Xe}-132$ & Nd-147 & Pu-238* \\
\hline $\mathrm{Zr}-93$ & $\mathrm{Xe}-135^{*}$ & Sm-147 & Pu-239* \\
\hline $\mathrm{Z}-94$ & Xe-136 & Sm-149 & Pu-240" \\
\hline Zr-95 & Cs-133* & Sm-150 & Pu-241* \\
\hline $\mathrm{Nb}-94$ & Cs-134 & Sm-151 & Pu-242* \\
\hline Tc-99 & Cs-135 & Sm-152 & Am-241* \\
\hline Rh-103 & Cs-137 & Gd-155 & Am-241m \\
\hline Rh-105 & Ba-136 & Eu-153 & Am-243* \\
\hline Ru-10! & La-139 & Eu-154 & $\mathrm{Cm}-242^{*}$ \\
\hline Ru-106 & Pr-141 & Eu-155 & $\mathrm{Cm}-243^{*}$ \\
\hline Pd-105 & Pr-143 & U-234* & $\mathrm{Cm}-244^{\circ}$ \\
\hline Pd-108 & Ce-144 & U-235* & \\
\hline
\end{tabular}

-Automatically included by SAS2H.

The lower burnup (18,000 MWd/MTU) cases were depleted for two cycles of 240 days. The higher burnup (25,000 MWd/MTU) cases were depleted for three cycles of 222.22 days. The specific power level for all cases was $17.514 \mathrm{MW}$ per assembly. There was no downtime between cycles and a five-jear downtime after the final cycle in all cases.

The resulting isotopics at the end of the five-year cooling period are listed in Tables 4-9. These were the values used in the criticality calculations described below. The SAS2H input decks are attached in Appendix A. 
Table 4. Reference isotopic set 1 3.0 ut \%. 18000 MWdRTU*

\begin{tabular}{lccc}
\hline Nuclide & $\begin{array}{c}\text { Number } \\
\text { density }\end{array}$ & Nuclide & $\begin{array}{c}\text { Number } \\
\text { density }\end{array}$ \\
\hline U-234 & $4.5843 E-06$ & Rh-103 & $1.5864 E-05$ \\
U-235 & $3.3960 E-04$ & Cs-133 & $2.8059 E-05$ \\
U-236 & $6.6971 E-05$ & Cs-135 & $7.7769 E-06$ \\
U-238 & $2.2205 E-02$ & Nd-143 & $2.0929 E-05$ \\
Pu-238 & $8.8353 E-07$ & Nd-145 & $1.5484 E-05$ \\
Pu-239 & $1.1460 E-04$ & Sm-147 & $5.3314 E-06$ \\
Pu-240 & $2.8115 E-05$ & Sm-149 & $1.5299 E-07$ \\
Pu-241 & $1.3620 E-05$ & Sm-150 & $6.1460 E-06$ \\
Pu-242 & $2.7445 E-06$ & Sm-151 & $4.2377 E-07$ \\
Am-241 & $3.8661 E-06$ & Sm-152 & $2.7537 E-06$ \\
O & $4.6455 E-02$ & Eu-153 & $1.7946 E-06$ \\
Mo-95 & $2.5875 E-05$ & Gd-155 & $1.0026 E-07$ \\
Tc-99 & $2.5634 E-05$ & & \\
\hline
\end{tabular}

-Megawatt days/metric ton initial uranium.

Table 5. Reference isotopic set 2

3.0 wt \%, $25000 \mathrm{MWd}_{\mathrm{MTU}}$

\begin{tabular}{|c|c|c|c|}
\hline Nuclide & $\begin{array}{l}\text { Number } \\
\text { density }\end{array}$ & Nuclide & $\begin{array}{l}\text { Number } \\
\text { density }\end{array}$ \\
\hline U-234 & 4.1151E-06 & Rh-103 & $2.1252 \mathrm{E}-05$ \\
\hline U-235 & $2.4980 \mathrm{E}-04$ & Cs-133 & 3.7614E-05 \\
\hline $\mathrm{U}-2.36$ & 8.0670E-05 & Cs-135 & $1.0747 \mathrm{E}-05$ \\
\hline U-238 & $2.2071 E-02$ & Nd-143 & $2.6524 \mathrm{E}-05$ \\
\hline Pu-238 & 1.9672E-(16 & Nd. 145 & $2.0563 \mathrm{E}-05$ \\
\hline Pu-239 & $1.2743 \mathrm{E}-04$ & Sm-147 & $6.4862 \mathrm{E}-06$ \\
\hline Pu-240 & $3.9900 \mathrm{E}-05$ & $\mathrm{Sm}-149$ & $1.5980 \mathrm{E}-07$ \\
\hline Pu-241 & 2.0457E-05 & $\mathrm{Sm} \cdot 150$ & $8.9709 E-06$ \\
\hline $\mathrm{Pu}-242$ & $6.2056 \mathrm{~F}-06$ & $\mathrm{Sm}-151$ & 4.9181E-07 \\
\hline Am-241 & 5.9277 E- 06 & $\mathrm{Sm}-152$ & $3.7235 E-06$ \\
\hline 0 & 4.6455E-02 & Eu-153 & 2.8184E-06 \\
\hline Mo.95 & 3.4761)E-05 & Gd-155 & $1.7613 \mathrm{E}-07$ \\
\hline Tc-99 & $3.4462 E-05$ & & \\
\hline
\end{tabular}

-Megawall days/metric ton initial uranium. 
Table 6. Reference isotopic set 3

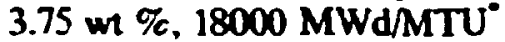

\begin{tabular}{lccc}
\hline Nuclide & $\begin{array}{c}\text { Number } \\
\text { density }\end{array}$ & Nuclide & $\begin{array}{c}\text { Number } \\
\text { density }\end{array}$ \\
\hline U-234 & $5.4708 E-06$ & Rh-103 & $1.5439 E-05$ \\
U-235 & $4.8322 E-04$ & Cs-133 & $28295 E-05$ \\
U-236 & $7.6752 E-05$ & Cs-135 & $9.1769 E-05$ \\
U-238 & $2.2057 E-02$ & Nd-143 & $21890 E-05$ \\
Pu-238 & $8.0056 E-07$ & Nd-145 & $1.5817 E-05$ \\
Pu-239 & $1.1618 E-04$ & Sm-147 & $5.5518 E-06$ \\
Pu-240 & $2.4356 E-05$ & Sm-149 & $1.7037 E-07$ \\
Pu-241 & $1.1818 E-05$ & Sm-150 & $6.0561 E-06$ \\
Pu-242 & $1.9009 E-06$ & Sm-151 & $4.8345 E-07$ \\
Am-241 & $.3 .3564 E-06$ & Sm-152 & $2.6548 E-06$ \\
O & $4.6458 E-02$ & Eu-153 & $1.6704 E-06$ \\
Mo-95 & $2.6362 E-05$ & Gd-155 & $9.3242 E-08$ \\
Tc-99 & $2.5850 E-05$ & & \\
\hline
\end{tabular}

Megawatt days/metric ton initial uranium.

Table 7. Reference isotopic set 4 3.75 w \%, 25000 MWdMTU

\begin{tabular}{lccc}
\hline Nuclide & $\begin{array}{l}\text { Number } \\
\text { density }\end{array}$ & Nuclide & $\begin{array}{c}\text { Number } \\
\text { density }\end{array}$ \\
\hline U-234 & 4.9660 E-06 & Rh-103 & $2.0758 E-05$ \\
U-235 & $3.7448 E-04$ & CS-133 & $3.8065 E-05$ \\
U-236 & $9.4302 E-05$ & Cs-135 & $1.2603 E-05$ \\
U-238 & $2.1935 E-02$ & Nd-143 & $2.8152 E-05$ \\
Pu-238 & $1.8076 E-06$ & Nd-145 & $2.1125 E-05$ \\
Pu-239 & $1.3180 E-04$ & Sm-147 & 6.8490 E-06 \\
Pu-240 & $3.5530 E-05$ & Sm-149 & $1.7808 E-07$ \\
P11-241 & $1.8700 E-05$ & Sm-150 & $8.8190 E-06$ \\
Pu-242 & $4.5287 E-06$ & Sm-151 & $5.5383 E-07$ \\
Am-241 & $5.4293 E-06$ & Sm-152 & $3.6210 E-06$ \\
O & $4.6458 E-02$ & Eu-153 & $2.6441 E-06$ \\
Mo-95 & $3.5562 E-05$ & Gd-155 & $1.6162 E-07$ \\
Tc-99 & $3.4863 E-05$ & & \\
\hline
\end{tabular}

Megawalt days/metric ton initial uranium 
Table 8. Reference isotopic set 5

4.5 wt \%, 18000 MWd/MTU $^{\circ}$

\begin{tabular}{lccc}
\hline Nuclide & $\begin{array}{l}\text { Number } \\
\text { density }\end{array}$ & Nuclide & $\begin{array}{l}\text { Number } \\
\text { density }\end{array}$ \\
\hline U-234 & $6.8134 E-06$ & Rh-103 & $1.5141 E-05$ \\
U-235 & $6.3569 E-04$ & Cs-133 & $2.8480 E-05$ \\
U-236 & $8.3957 E-05$ & Cs-135 & $1.0523 E-05$ \\
U-238 & $2.1901 E-02$ & Nd-143 & $2.2600 E-05$ \\
Pu-238 & $7.2349 E-07$ & Nd-145 & $1.6059 E-05$ \\
Pu-239 & $1.1687 E-04$ & Sm-147 & $5.7241 E-06$ \\
Pu-240 & $2.1413 E-05$ & Sm-149 & $1.8366 E-07$ \\
Pu-241 & $1.0316 E-05$ & Sri-150 & $6.0269 E-06$ \\
Pu-242 & $1.3715 E-06$ & Sm-151 & $5.4511 E-07$ \\
Am-241 & $2.9311 E-06$ & Sm-152 & $25670 E-06$ \\
O & $4.6462 E-02$ & Eu-153 & $1.5715 E-06$ \\
Mo-95 & $2.6712 E-05$ & Gd-155 & $8.8526 E-08$ \\
Tc-99 & $26015 E-05$ & & \\
\hline
\end{tabular}

Megawatt days/metric ton initial uranium.

Table 9. Reference isotopic set 6 4.5 wt \%. $25000 \mathrm{MWd}^{\mathrm{MTU}}$

\begin{tabular}{llll}
\hline Nuclide & $\begin{array}{l}\text { Number } \\
\text { density }\end{array}$ & Nuclide & $\begin{array}{l}\text { Number } \\
\text { density }\end{array}$ \\
\hline U-234 & 6.2365E-06 & Rh-103 & 2.0397E-05 \\
U-235 & 5.1213E-04 & Cs-133 & $3.8420 E-05$ \\
U-236 & $1.0479 E-04$ & Cs-135 & $1.4418 E-05$ \\
U-238 & $2.1790 E-02$ & Nd-143 & $2.9402 E-05$ \\
Pu-238 & $1.6481 E-06$ & Nd-145 & $2.1543 E-05$ \\
Pu-239 & $1.3503 E-04$ & Sm-147 & $7.1435 E-06$ \\
Pu-240 & $3.1871 E-05$ & Sm-149 & $1.9093 E-07$ \\
Pu-241 & $1.7023 E-05$ & Sm-150 & $\mathbf{8 . 8 3 6 3 E - 0 6}$ \\
Pu-242 & $3.3969 E-06$ & Sm-151 & $6.2147 E-07$ \\
Am-241 & $4.9501 E-06$ & Sm-152 & $3.5323 E-06$ \\
O & $4.6462 E-02$ & Eu-153 & $2.4987 E-06$ \\
Mo-95 & $\mathbf{3 . 6 1 5 8 E - 0 5}$ & Gd-155 & $1.5066 E-07$ \\
Tc-99 & $3.5176 E-05$ & & \\
\hline
\end{tabular}

- Mcgawatt days/metric ton initial uranium. 


\section{CRIICALITY BENCHMARK CALCULATIONS}

The reference problem set of fresh fuel critical experiments from ref. 1 is listed and briefly described in Table 10. More complete descriptions may be found in the individual references listed in the table. The last experiment listed in the table was not used since it was a mixed-oxide experiment intended to simulate an assembly with 20,000 MWd/MTU burnup. The other 15 problems were analyzed with each of the 6 sets of spent fuel isotopics for a total of 90 cases. The CSAS4 input deiks used to model the experiments in ref. 1 were modified by commenting out the actual fresh fuel isotopics and inserting one set of the spent fuel isotopics shown in Tables 4 through 9. Copies of the input decks for the 15 benchmark problems are provided in Appendix B. Note that the fresh fuel isotopics are commented out, and comments show where the spent fuel isotopics are to be inserted and what mixture number should be assigned. The results for all 90 cases are presented in Table 11. Each case used 600 neutrons per generation for 205 generations, except as noted otherwise in the table. 
Table 10. Fresh fuel critical experiments for burnup credit calculations

\begin{tabular}{|c|c|c|}
\hline Experimental assembly & $\begin{array}{l}\text { Experiment } \\
\text { reference } \\
\text { numbers }\end{array}$ & Data source \\
\hline $\begin{array}{l}2 \times 2 \text { rectangular array of water-moderated and } \\
\text { reflected fuel units separated by neutron flux tra } s\end{array}$ & $214 R$ & $\begin{array}{l}\text { S. R. Bierman, } \\
\text { PNL-7167, } 1990 \text { (ref. 5) }\end{array}$ \\
\hline $\begin{array}{l}2 \times 2 \text { rectangular array of water-moderated and } \\
\text { reflected fuel units separated by neutmn flux traps } \\
\text { containing voids }\end{array}$ & $214 V 3$ & $\begin{array}{l}\text { S. R. Bierman, } \\
\text { PNL-7167, } 1990 \text { (ref. 5) }\end{array}$ \\
\hline $\begin{array}{l}\text { Three rectangular water-moderated and reflected } \\
\text { fuel units in a row separated by water gaps } \\
\text { containing plates of either boral, steel, or } \\
\text { aluminum }\end{array}$ & $\begin{array}{l}005 \\
017 \\
028 \\
024\end{array}$ & $\begin{array}{l}\text { S. R. Bierman et al., } \\
\text { PNL-2438, } 1977 \text { (ref. 6) }\end{array}$ \\
\hline $\begin{array}{l}\text { Three separate water-moderated and reflected } \\
\text { rectangular fuel units in a row with lead, depleted } \\
\text { uranium, or steel walls on either side }\end{array}$ & a & $\begin{array}{l}\text { S. R. Bierman et al., } \\
\text { PNL-3926, } 1981 \text { (ref. 7) } \\
\text { S. R. Bierman et al., } \\
\text { PNL-3602, } 1981 \text { (ref. 8) }\end{array}$ \\
\hline $\begin{array}{l}3 \times 3 \text { rectangular array of water-moderated and } \\
\text { reflected fuel units separated by water gaps } \\
\text { containing equally spaced } B_{4} C \text { rods }\end{array}$ & 2282 & $\begin{array}{l}\text { M. N. Baldwin et al., } \\
\text { BAW-1484-7, } 1979 \\
\text { (ref. 9) }\end{array}$ \\
\hline $\begin{array}{l}\text { Slightly undermoderated assemblies of fuel rods } \\
\text { moderated with water containing identical } \\
\text { concentrations of dissolved boron }\end{array}$ & $\begin{array}{l}173 \\
177 \\
178 \\
181\end{array}$ & $\begin{array}{l}\text { B. M. Durst et al., } \\
\text { PNL-4267, } 1982 \text { (ref. 10) }\end{array}$ \\
\hline $\begin{array}{l}\text { Seven rectangular units of fuel rods encased in } \\
\text { AB. alloy sleeves arranged in a subcritical } \\
\text { shipping cask geometry, water-moderated and } \\
\text { reflected }\end{array}$ & TTC-5 & $\begin{array}{l}\text { S. R. Bierman } \\
\text { PNL-6838, } 1989 \text { (ref. 11) }\end{array}$ \\
\hline $\begin{array}{l}\text { A water-moderated and reflected assembly of } \\
\mathrm{PuO}_{2}-\mathrm{UO}_{2} \text { fuel rods and } \mathrm{UO}_{2} \text { fucl rods arranged in } \\
\text { a uniform pattern :0 arnroximate an assembly } \\
\mathrm{Pu} /{ }^{\mathfrak{m}} \mathrm{U} \text { ratio characteristic of } 20,000 \mathrm{MWd} / \mathrm{MTU} \\
\text { burnup }\end{array}$ & 196 & $\begin{array}{l}\text { S. R. Bicrman ct al., } \\
\text { PNL-4976, } 1982 \text { (ref. 12) }\end{array}$ \\
\hline
\end{tabular}

- No reference number given. Assembly with $19.56 \mathrm{~mm}$ between fuel and reflecting walls.

- Boron concentration such that assembly with closer-spaced fuel rods has the smaller critical mass.

- 1264 fucl rods and $k_{\text {efl }}$ of 0.92 .

- Measured subcritical $k_{\text {efl }}$ values also provided as funclion of Gd concentration is the moderalor. 
Table 11. Reference benchmark results using spent fuel isotopics

\begin{tabular}{|c|c|c|c|c|c|c|}
\hline Experiment & $\begin{array}{l}3.0 \text { wt } \% \\
18 \mathrm{GWd} / \mathrm{MTU}\end{array}$ & $\begin{array}{c}3.0 \mathrm{wt} \% \\
25 \mathrm{GWd} / \mathrm{MTU}\end{array}$ & $\begin{array}{c}3.75 \text { wt } \% \\
18 \mathrm{GWd} / \mathrm{MTU}\end{array}$ & $\begin{array}{c}3.75 \text { wt \% } \\
25 \mathrm{GWd} / \mathrm{MTU}\end{array}$ & $\begin{array}{c}4.5 \mathrm{wt} \% \\
18 \mathrm{GWd} / \mathrm{MTU}\end{array}$ & $\begin{array}{c}4.5 \mathrm{wt} \% \\
25 \mathrm{GWd} / \mathrm{MTU}\end{array}$ \\
\hline $214 R$ & $0.79706(203)^{\bullet}$ & $0.73890(196)$ & $0.83495(195)$ & $0.79795(174)$ & $0.87645(194)$ & $57(188)$ \\
\hline $214 V 3$ & $0.79061(182)$ & $0.74729(\cdot 74)$ & $0.83900(18.3)$ & $0.79622(i 83)$ & $0.87667(193)$ & $0.83357(18.3)$ \\
\hline 2282 & $0.88796(199)$ & $0.83402(: 66)$ & $0.93683(188)$ & $0.89251(200)$ & $0.98586(198)$ & $0.93913(187)$ \\
\hline 5 & $0.91761)(188)$ & $0.86904(177)$ & $0.98218(202)$ & $0.93404(186)$ & $1.03204(189)$ & $0.98566(207)$ \\
\hline 17 & $0.921965(1$ & $0.86654(167)$ & $0.97958(191)$ & & 1.03066 & $0.98659(194)$ \\
\hline 24 & (184) & 0.8686 & 0.981 & & & $74(192)$ \\
\hline 028 & 2) & 172) & 0.9 & & 76) & $8(193)$ \\
\hline $\begin{array}{l}\text { PNL-360)2 } \\
\text { (steel walls) }\end{array}$ & $0.79743(193)$ & $0.75216(167)$ & $0.84587(183)$ & $0.80181(184)$ & $0.88688(210)$ & $0.84010(185)$ \\
\hline $\begin{array}{l}\text { PNL-3926 } \\
\text { (U walls) }\end{array}$ & $0.80602(174)$ & $0.76228(190)$ & $0.84818(188)$ & $0.80746(166)$ & $0.88562(173)$ & $0.84502(176)$ \\
\hline $\begin{array}{l}\text { PNL-3926 } \\
\text { (lead walls) }\end{array}$ & $0.79899(187)$ & $0.755 .39(164)$ & $0.84455(184)$ & $0.80299(200)$ & $0.88064(194)$ & $0.84121(187)$ \\
\hline 173 & $0.791 .32(249)^{b}$ & $0.74769(276)^{b}$ & $0.83815(288)^{b}$ & $0.79589(261)^{b}$ & $(259)^{b}$ & $0.84068(31.3)^{b}$ \\
\hline 177 & $0.77227(123)$ & $0.72487(135)$ & $0.83253(142)$ & $0.78994(160)$ & $0.88162(144)$ & $0.84069(147)$ \\
\hline 178 & & $0.74583(273)^{6}$ & $0.8304^{2}(278)^{b}$ & $0.78859(240)^{b}$ & $0.86806(249)^{b}$ & $0.82301(282)^{h}$ \\
\hline 181 & & & & & & $0.82120(162)$ \\
\hline TTC-5 & $0.71351(189)$ & $0.67805(172)$ & $0.76147(192)$ & $0.721 .3(180)$ & c..90058(211) & $0.76501(217)$ \\
\hline
\end{tabular}

- Read as $0.797(16 \pm 0.0020 .3$.

b $(X)$ neutrons per generation for 105 generations. 


\section{CONCLUSIONS}

The reference benchmark calculations in Table 11 provide a means for benchmarking different calculational methods and provides users with a reference set of criticality problems at six different enrichment/burnup combinations. This should aid in addressing some of the computer code validation concerns regarding burnup credit.

\section{REFERENCES}

1. S. M. Bowman, Validation of SCALE-4 for a Reference Problem Set, ORNLM-1332, Martin Marietta Energy Systems, Inc., Oak Ridge Natl. Lab. (November 1990).

2. C. V. Parks, Ed., SCALE: A Modular Code System for Performing Standardized Computer Analyses for Licensing Evaluation, NUREG/CR-0200, Rev. 4 (ORNLNUREG/CSD-2/R4), Vols. I, II, and III (Draft February 1990).

3. O. W. Hermann et al., "SAS2H: A Coupled One-Dimensional Depletion and Shielding Analysis Code." Sect. S2 of NUREG/CR-0200. Rev. 4 (ORNL/NUREG/CSD-2/R4), Vol. I (Dran February 1990).

4. N. F. Landers et al., "CSAS4: An Enhanced Criticality Safety Analysis Module with an Optimum Pitch Search Option," Sect. C4 of NUREG/CR-0200, Rev. 4. Vol. 1 (Draft February 1990).

5. S. R. Bierman, Criticality Experiments $W_{i}^{\prime 2}$. Neutron Flux Traps Containing Voids, PNL-7167, Battelle Pacific Northwest Laboratory (April 1990).

6. S. R. Bierman et al.. Critical Separation Between Subcritical Clusters of $2.35 \mathrm{Wl}^{\circ}{ }^{\circ}{ }^{23 s} \mathrm{U}$ Enriched $\mathrm{UO}_{2}$ Rods in Water With Fixed Neutron Poisons, PNL-24.38, Battelle Pacific Northwest Laboratory (October 1977).

7. S. R. Bierman et al.. Criticaliny Experiments with Subcritical Clusters of $2.35 W t \%$ and 4.31 $\mathrm{Wl}_{\mathrm{t}}{ }^{235} \mathrm{U}$ Enviched $\mathrm{UO}_{2}$ Rods in Water with Uranium or Lead Reflecting Walls, PNL-3926, Battelle Pacific Northwest Laboratory (December 1981).

8. S. R. Bierman and E. D. Clayton, Criticality Experiments with Subcritical Clusters of $2.35 \mathrm{~W} \% \mathrm{~F}$ and $4.31 \mathrm{~W} \mathrm{~W}_{\mathrm{c}}{ }^{235} \mathrm{U}$ Enriched $\mathrm{UO}_{2}$ Rods in Water with Steel Reflecting Walls. PNL-3602. Baltelle Pacific Northwest Laboratory (April 1981).

9. M. N. Baldwin el al.. Critical Experiments Supponing Close Proximit! Water Storage of Power Reacior Fuel, BAW-1484.7. Babcock and Wilcox (July 1979). 
10. B. M. Durst et al. Critical Experiments with 4.31 Wt $\%_{c}{ }^{23} U$ Enriched $\mathrm{UO}_{2}$ Rads in Highly Borated Water Lattices. PNL-4267. Batte!le Pacific Northwest Labura:ory (August 1982).

11. S. R. Bierman. Reactivity Measurements on an Expenimental Assembly of 4.31 Hicic ${ }^{215} \mathrm{U}$ Enriched $\mathrm{UO}_{2}$ Fuel Rods Arranged in a Shipping Cask Geometn. PNL-6838. Battelle Pacific Northwest Laboratory (October 1989).

12. S. R. Bierman et al., Criticaliny Ex, reriments with Low Enriched $\mathrm{UO}_{2}$ Fuel Rods in Water Containing Dissolved Gadolnium, PNL-4976, Battelle Pacific Northwest Laboratory (February 1984). 
APPENDIX A 
SAS2H INPUT DECK FOR 3.00 WT' 18000 MWD/T DEPLETION

\begin{tabular}{|c|c|c|c|c|c|c|c|c|c|c|}
\hline $\begin{array}{l}=\text { SAS } 2 H \\
\text { BURNUP CF } \\
27 \text { BURNUPI }\end{array}$ & $\begin{array}{l}\text { RED } \\
\text { LIB }\end{array}$ & PA & $\begin{array}{r}R M=(H A I \\
-3.00 \\
\text { LATTICE }\end{array}$ & $\begin{array}{l}\text { LT02, } \mathrm{S} \\
\text { D WT } \\
\text { ECELL }\end{array}$ & $\begin{array}{r}\text { SKIPSHI } \\
\text { 18GWD }\end{array}$ & $\begin{array}{l}\text { PDATA } \\
/ T \quad \text { DI }\end{array}$ & PLET & ON & & \\
\hline $\mathrm{UO2}_{96.9608^{1}}^{1}$ & $\begin{array}{l}.9 \\
\text { EN }\end{array}$ & & 811922 & 234. & 0254 & 92235 & 3.0 & 92236 & .0138 & 92238 \\
\hline KR-85 & 1 & 0 & $1-20$ & 811 & END & & & & & \\
\hline SR-90 & 1 & 0 & $1-20$ & 811 & END & & & & & \\
\hline$Y-89$ & 1 & 0 & $1-20$ & 811 & END & & & & & \\
\hline MO-95 & 1 & 0 & $1-20$ & 811 & END & & & & & \\
\hline ZR-93 & 1 & 0 & $1-20$ & 811 & END & & & & & \\
\hline ZR-94 & 1 & 0 & $1-20$ & 811 & END & & & & & \\
\hline ZR-95 & 1 & 0 & $1-20$ & 811 & END & & & & & \\
\hline NB-94 & $\overline{1}$ & 0 & $1-20$ & 811 & END & & & & & \\
\hline TC-99 & 1 & 0 & $1-20$ & 811 & END & & & & & \\
\hline RH-103 & 1 & 0 & $1-20$ & 811 & END & & & & & \\
\hline RH-105 & 1 & 0 & $1-20$ & 811 & END & & & & & \\
\hline RU-101 & 1 & 0 & $1-20$ & 811 & END & & & & & \\
\hline RU-106 & 1 & 0 & $1-20$ & 811 & END & & & & & \\
\hline PD-.105 & 1 & 0 & $1-20$ & 811 & END & & & & & \\
\hline PD-108 & 1 & 0 & $1-20$ & 811 & END & & & & & \\
\hline AG-109 & 1 & 0 & $1-20$ & 811 & END & & & & & \\
\hline SB-124 & 1 & 0 & $1-20$ & 811 & END & & & & & \\
\hline$X E-131$ & 1 & 0 & $1-20$ & 811 & END & & & & & \\
\hline$Y E-132$ & 1 & 0 & $1-20$ & 811 & END & & & & & \\
\hline$X E-135$ & 2 & 0 & $6.72-9$ & 9811 & 1 END & & & & & \\
\hline$X E-136$ & 1 & 0 & $1-20$ & 811 & END & & & & & \\
\hline$C S-134$ & 1 & 0 & $1-20$ & 811 & END & & & & & \\
\hline$C S-135$ & 1 & 0 & $1-20$ & 811 & END & & & & & \\
\hline$c 5-137$ & 1 & 0 & $1-20$ & 811 & END & & & & & \\
\hline$B A-136$ & 1 & 0 & $1-20$ & 811 & END & & & & & \\
\hline LA-139 & 1 & 0 & $1-20$ & 811 & END & & & & & \\
\hline PR-141 & 1 & 0 & $1-20$ & 811 & END & & & & & \\
\hline PR-143 & 1 & 0 & $1-20$ & 811 & END & & & & & \\
\hline$C E-144$ & 1 & 0 & $1-20$ & 811 & END & & & & & \\
\hline ND-143 & 1 & 0 & $1-20$ & 811 & END & & & & & \\
\hline$N D-145$ & 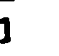 & 0 & $1-20$ & 811 & END & & & & & \\
\hline PM-147 & 1 & 0 & $1-20$ & 811 & END & & & & & \\
\hline ND -147 & 1 & 0 & $1-20$ & 811 & END & & & & & \\
\hline$S M-147$ & 1 & 0 & $1-20$ & 811 & END & & & & & \\
\hline$S M-149$ & 1 & 0 & $1-20$ & 811 & END & & & & & \\
\hline$S M-150$ & 1 & 0 & $1-20$ & 811 & END & & & & & \\
\hline$S M-151$ & 1 & 0 & $1-20$ & 811 & END & & & & & \\
\hline SM-152 & 1 & 0 & $1-20$ & 811 & END & & & & & \\
\hline GD-155 & 1 & 0 & $1-20$ & 811 & END & & & & & \\
\hline$E U-153$ & 1 & 0 & $1-20$ & 811 & END & & & & & \\
\hline$E U-154$ & 1 & 0 & $1-20$ & 811 & END & & & & & \\
\hline$E U-155$ & $\overline{1}$ & 0 & $1-20$ & 811 & END & & & & & \\
\hline ZIRCALLO & & 2 & 1620 & END & & & & & & \\
\hline $\mathrm{H2O}$ & & 3 & $\overline{D E N}=.73$ & 331 & & 570 & END & & & \\
\hline
\end{tabular}




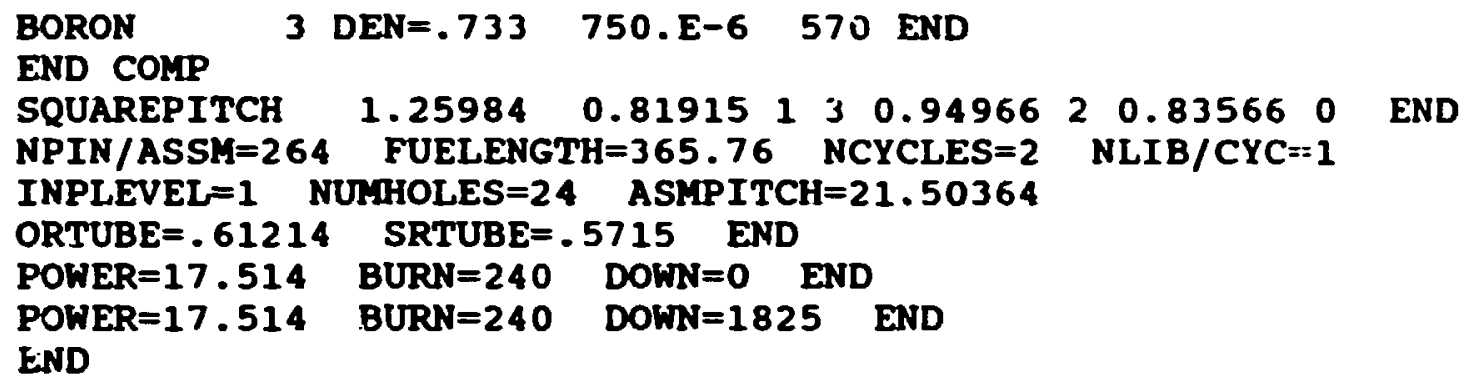




\begin{tabular}{|c|c|c|c|c|c|c|c|c|c|c|}
\hline $\begin{array}{l}=\text { SAS } \angle H \\
\text { BURNUP CI } \\
27 \text { BURNUPI }\end{array}$ & $\begin{array}{l}\text { REI } \\
\text { LIE }\end{array}$ & PA & $\begin{array}{r}\text { RY }=(\text { HAL } \\
-3.00 \\
\text { LATTICE }\end{array}$ & $\begin{array}{l}\text { LTO3, } \\
0 \text { WT: } \\
\text { ECELL }\end{array}$ & $\begin{array}{r}\text { SKIPSHI } \\
25 \text { GWT }\end{array}$ & $\begin{array}{l}\text { [PDATA) } \\
\mathrm{T} \text { D DI }\end{array}$ & EPLET & ON & & \\
\hline${ }_{962}^{\mathrm{U} 2}{ }^{1}{ }^{1}$ & E & $\begin{array}{l}5 \\
\text { ID }\end{array}$ & 811922 & 234 & .0254 & 92235 & 3.0 & 92236 & .0138 & 92238 \\
\hline $\mathrm{KR}-85$ & 1 & 0 & $1-20$ & 811 & END & & & & & \\
\hline SR-90 & 1 & 0 & $1-20$ & 811 & END & & & & & \\
\hline$Y=89$ & 1 & 0 & $1-20$ & 811 & END & & & & & \\
\hline MO-95 & 1 & $\mathbf{0}$ & $1-20$ & 811 & END & & & & & \\
\hline $2 R-93$ & 1 & 0 & $1-20$ & 811 & END & & & & & \\
\hline $2 R-94$ & 1 & $\mathbf{0}$ & $1-20$ & 811 & END & & & & & \\
\hline ZR-55 & 1 & 0 & $1-20$ & 811 & END & & & & & \\
\hline NB-94 & 1 & 0 & $1-20$ & 811 & END & & & & & \\
\hline TC-99 & 1 & 0 & $1-20$ & 811 & END & & & & & \\
\hline RH-103 & 1 & $\mathbf{0}$ & $1-20$ & 811 & END & & & & & \\
\hline RH-105 & 1 & $\mathbf{0}$ & $1-20$ & $81 i$ & END & & & & & \\
\hline RU-101 & 1 & $\mathbf{0}$ & $1 \cdot-20$ & 811 & END & & & & & \\
\hline RU-106 & 1 & $\mathbf{0}$ & $1-20$ & 811 & END & & & & & \\
\hline PD-105 & 1 & $\mathbf{0}$ & $1-20$ & 811 & END & & & & & \\
\hline PD-108 & 1 & 0 & $1-20$ & 811 & END & & & & & \\
\hline AG-109 & 1 & 0 & $1-20$ & 811 & END & & & & & \\
\hline SB-124 & 1 & 0 & $1-20$ & 811 & END & & & & & \\
\hline$X E-131$ & 1 & 0 & $1-20$ & 811 & END & & & & & \\
\hline$X E-132$ & 1 & 0 & $1-20$ & 811 & END & & & & & \\
\hline$X E-135$ & 1 & 0 & $6.72-9$ & 981 & 1 END & & & & & \\
\hline$X E-136$ & 1 & 0 & $1-20$ & 811 & END & & & & & \\
\hline$C S-134$ & 1 & 0 & $1-20$ & 811 & END & & & & & \\
\hline$C S-135$ & 1 & 0 & $1-20$ & 811 & END & & & & & \\
\hline CS-137 & 1 & 0 & $1-20$ & 811 & END & & & & & \\
\hline$B A-136$ & 1 & 0 & $1-20$ & 811 & END & & & & & \\
\hline LA- 139 & 1 & 0 & $1-20$ & 811 & END & & & & & \\
\hline PR-141 & 1 & 0 & $1-20$ & 811 & END & & & & & \\
\hline PR-143 & 1 & 0 & $1-20$ & 811 & END & & & & & \\
\hline$C E-144$ & 1 & 0 & $1-20$ & 811 & END & & & & & \\
\hline$N D-143$ & 1 & 0 & $1-20$ & 811 & END & & & & & \\
\hline$N D-145$ & 1 & 0 & $1-20$ & 811 & END & & & & & \\
\hline PM- 147 & 1 & 0 & $1-20$ & 811 & END & & & & & \\
\hline$N D-147$ & 1 & 0 & $1-20$ & 811 & END & & & & & \\
\hline $5 x-147$ & 1 & 0 & $1-20$ & 811 & END & & & & & \\
\hline$S H-149$ & 1 & 0 & $1-20$ & 811 & END & & & & & \\
\hline$S M-150$ & 1 & 0 & $1-20$ & 811 & END & & & & & \\
\hline$S M-151$ & 1 & 0 & $1-20$ & 811 & END & & & & & \\
\hline$S M-152$ & 1 & 0 & $1-20$ & 811 & END & & & & & \\
\hline GD- 155 & 1 & 0 & $1-20$ & 811 & END & & & & & \\
\hline$E U-153$ & 1 & 0 & $1-20$ & 811 & END & & & & & \\
\hline EU-154 & 1 & 0 & $1-20$ & 811 & END & & & & & \\
\hline EU-155 & 1 & 0 & $1-20$ & 811 & END & & & & & \\
\hline 2IRCALLO & & 2 & 1620 & END & & & & & & \\
\hline $\mathrm{H} 2 \mathrm{O}$ & & 3 & $D E N=.73$ & 331 & 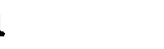 & 570 & END & & & \\
\hline
\end{tabular}




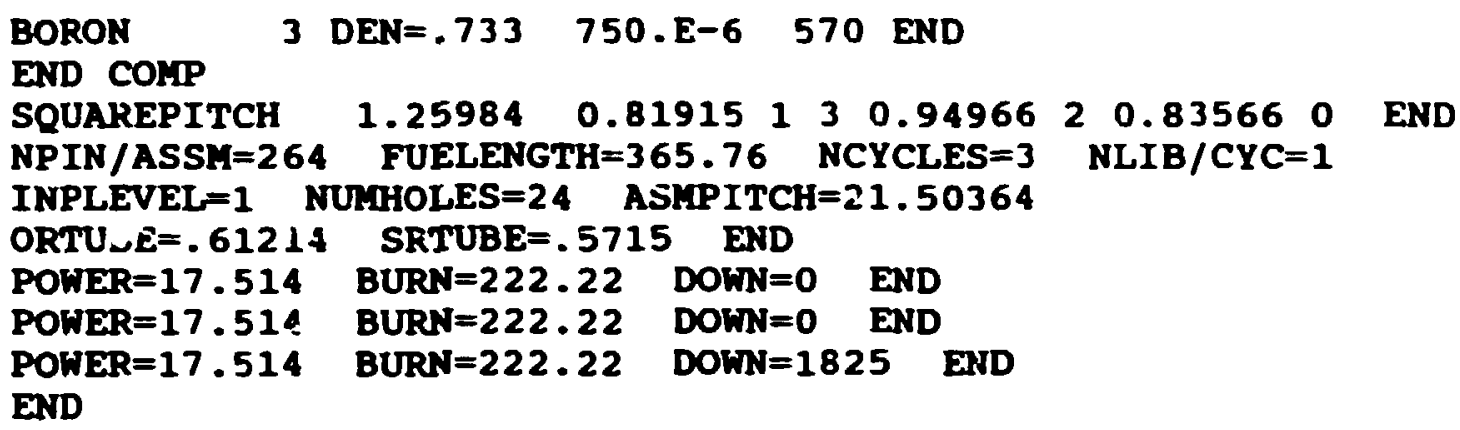


SAS2H INPUT DECK FOR 3.75 WT 18000 MWD/T DEPLETION

\begin{tabular}{|c|c|c|c|c|c|c|c|c|c|c|}
\hline $\begin{array}{l}=\text { SAS } 2 \mathrm{H} \\
\text { BURNUP CI } \\
27 \text { BURNUPI }\end{array}$ & $\begin{array}{l}\text { RED } \\
\text { LIB }\end{array}$ & $\begin{array}{l}\text { PA } \\
\text { DIT } \\
8\end{array}$ & $\begin{array}{r}\text { RH }=(\text { HAL } \\
-3.75 \\
\text { LATTICE }\end{array}$ & $\begin{array}{l}\text { LTO2, } \\
5 \text { WT } \\
\text { ECELL }\end{array}$ & $\begin{array}{r}\text { SKIPSHI } \\
\text { I8GWL }\end{array}$ & $\begin{array}{l}\text { PDA } F A] \\
/ T \quad D I\end{array}$ & PLETI & ON & & \\
\hline $\begin{array}{l}\text { UO2 } \\
96.1973^{1}\end{array}$ & EN & & 811922 & $23 i$ & 0294 & 92235 & 3.75 & 92236 & .0233 & 92238 \\
\hline $\mathrm{KR}-85$ & 1 & 0 & $1-20$ & 811 & END & & & & & \\
\hline SR-90 & 1 & 0 & $1-20$ & 311 & END & & & & & \\
\hline$Y-89$ & 1 & 0 & $1-20$ & 811 & END & & & & & \\
\hline 110-95 & 1 & 0 & $1-20$ & 811 & END & & & & & \\
\hline ZR-93 & 1 & 0 & $1-20$ & 811 & END & & & & & \\
\hline ZR-94 & 1 & 0 & $1-20$ & 811 & END & & & & & \\
\hline ZR-95 & 1 & 0 & $1-20$ & 811 & END & & & & & \\
\hline$N B-94$ & 1 & 0 & $1-20$ & 811 & END & & & & & \\
\hline TC-99 & 1 & 0 & $1-20$ & 811 & END & & & & & \\
\hline RH-103 & 1 & 0 & $1-20$ & 811 & END & & & & & \\
\hline RH-105 & 1 & 0 & $1-20$ & 811 & END & & & & & \\
\hline RU-101 & 1 & 0 & $1-20$ & 811 & END & & & & & \\
\hline RU-106 & : & 0 & $1-20$ & 811 & END & & & & & \\
\hline PD-105 & 1 & 0 & $1-20$ & 811 & END & & & & & \\
\hline PD- 108 & 1 & 0 & $1-20$ & 811 & END & & & & & \\
\hline AG-109 & 1 & 0 & $1-20$ & 811 & ENC & & & & & \\
\hline SB-124 & 1 & $\mathbf{0}$ & $1-20$ & 811 & END & & & & & \\
\hline$X E-131$ & 1 & 0 & $1-20$ & 811 & END & & & & & \\
\hline$X E-132$ & 1 & 0 & $1-20$ & 811 & END & & & & & \\
\hline$X E-135$ & 1 & 0 & $6.72-9$ & 9811 & 1 END & & & & & \\
\hline$X E-136$ & 1 & 0 & $1-20$ & 811 & END & & & & & \\
\hline$c s-134$ & 1 & 0 & $1-20$ & 811 & END & & & & & \\
\hline$C s-135$ & 1 & 0 & $1-20$ & 811 & END & & & & & \\
\hline $\operatorname{cs}-137$ & 1 & 0 & $1-20$ & 811 & END & & & & & \\
\hline$B A-136$ & 1 & 0 & $1-20$ & 811 & END & & & & & \\
\hline$L A-139$ & 1 & 0 & $1-20$ & 811 & END & & & & & \\
\hline PR-141 & 1 & 0 & $1-20$ & 811 & END & & & & & \\
\hline PR-143 & 1 & 0 & $1-20$ & 811 & END & & & & & \\
\hline$C E-144$ & 1 & 0 & $1-20$ & 811 & END & & & & & \\
\hline$N D-143$ & 1 & 0 & $1-20$ & 811 & END & & & & & \\
\hline ND - 145 & 1 & 0 & $1-20$ & 811 & END & & & & & \\
\hline$P M-147$ & 1 & 0 & $1-20$ & 811 & END & & & & & \\
\hline ND -147 & 1 & 0 & $1-20$ & 811 & END & & & & & \\
\hline $5 M-147$ & 1 & 0 & $1-20$ & 811 & END & & & & & \\
\hline$S M-149$ & 1 & 0 & $1-20$ & 81.1 & END & & & & & \\
\hline$S M-150$ & 1 & 0 & $1-20$ & 811 & END & & & & & \\
\hline SH-151 & 1 & 0 & $1-20$ & 811 & END & & & & & \\
\hline$S H-152$ & 1 & 0 & $1-20$ & 811 & END & & & & & \\
\hline GD-155 & 1 & 0 & $1-20$ & 811 & END & & & & & \\
\hline$E U-153$ & 1 & 0 & $1-20$ & 811 & END & & & & & \\
\hline$E U-154$ & 1 & 0 & $1-20$ & 811 & END & & & & & \\
\hline$E U-155$ & 1 & 0 & $1-20$ & 811 & END & & & & & \\
\hline ZIRCALLO & & 2 & 1620 & END & & & & & & \\
\hline $\mathrm{H} 2 \mathrm{O}$ & & 3 & $D E N=.73$ & 331 & & 570 & END & & & \\
\hline
\end{tabular}


BORON

3 DEN $=.733 \quad 750 . E-6 \quad 570$ END

END COMP

SQUAREPITCH $1.259840 .81915130 .9496620 .83566 \quad 0 \quad$ END

NDIN/ASSH $=264$ FUELENGTH $=365.76$ NCYCLES $=2$ NLIB $/$ CYC $=1$

INPLEVEL $=1$ NUMHOLES $=24$ ASMPITCH $=21.50364$

ORTUBE $=.61214$ SRTUBE $=.5715$ END

POWER $=17.514 \quad B U R N=240$ DOWN $=0$ END

POWER $=17.514 \quad B U R N=240$ DOWN $=1825$ END

END 


\begin{tabular}{|c|c|c|c|c|c|c|c|c|c|c|}
\hline $\begin{array}{l}=\text { =SAS2H } \\
\text { BURNUP C } \\
\text { 2.7BURNUP }\end{array}$ & $\begin{array}{l}\mathbf{R E} \\
\mathbf{L I}\end{array}$ & & $\begin{array}{r}R M=(H A \\
-3.7 \\
\text { LATTIC }\end{array}$ & $\begin{array}{l}\text { LTO3, } \\
5 \text { WT } \\
\text { ECELL }\end{array}$ & $\begin{array}{r}\text { SRIPSHI } \\
25 G W D\end{array}$ & $\begin{array}{l}\text { (PDATA) } \\
(T \quad \mathrm{DE}\end{array}$ & EPLETI & & & \\
\hline $\begin{array}{l}102 \\
96.1973^{1}\end{array}$ & & $\begin{array}{l}95 \\
\text { ND }\end{array}$ & 8119 & 234 & .0294 & 92235 & 3.75 & 92236 & .0233 & 92238 \\
\hline KR-85 & 1 & 0 & $1-20$ & 811 & END & & & & & \\
\hline SR-90 & 1 & 0 & $1-20$ & 811 & END & & & & & \\
\hline$Y-89$ & 1 & 0 & $1-20$ & 811 & END & & & & & \\
\hline MO-95 & 1 & 0 & $1-20$ & 811 & END & & & & & \\
\hline 2R-93 & 1 & 0 & $1-20$ & 811 & END & & & & & \\
\hline 2R-94 & 1 & 0 & $1-20$ & 811 & END & & & & & \\
\hline 2R-95 & 1 & 0 & $1-20$ & 811 & END & & & & & \\
\hline NB-94 & 1 & 0 & $1-20$ & 811 & END & & & & & \\
\hline TC-99 & 1 & 0 & $1-20$ & 811 & END & & & & & \\
\hline RH-103 & 1 & 0 & $1-20$ & 811 & END & & & & & \\
\hline RH-105 & 1 & 0 & $1-20$ & 811 & END & & & & & \\
\hline RU-101 & 1 & 0 & $1-20$ & 811 & END & & & & & \\
\hline RU-106 & 1 & 0 & $1-20$ & 811 & END & & & & & \\
\hline PD- 105 & 1 & 0 & $1-20$ & 811 & END & & & & & \\
\hline PD- 108 & 1 & 0 & $1-20$ & 811 & END & & & & & \\
\hline AG-109 & 1 & 0 & $1-20$ & 811 & END & & & & & \\
\hline SB- 124 & 1 & 0 & $1-20$ & 811 & END & & & & & \\
\hline$X E-131$ & 1 & 0 & $1-20$ & 811 & END & & & & & \\
\hline$x E-132$ & 1 & 0 & $1-20$ & 811 & END & & & & & \\
\hline$X E-135$ & 1 & 0 & $6.72-$ & $\begin{array}{ll}9 & 81\end{array}$ & 1 END & & & & & \\
\hline$X E-136$ & 1 & 0 & $1-20$ & 811 & END & & & & & \\
\hline CS-134 & 1 & 0 & $1-20$ & 811 & END & & & & & \\
\hline CS-I35 & 1 & 0 & $1-20$ & 811 & END & & & & & \\
\hline$C S-137$ & 1 & 0 & $1-20$ & 811 & END & & & & & \\
\hline$B A-136$ & 1 & 0 & $1-20$ & 811 & END & & & & & \\
\hline LA-139 & 1 & 0 & $1-20$ & 811 & END & & & & & \\
\hline PR-141 & 1 & 0 & $1-20$ & 811 & END & & & & & \\
\hline PR-143 & 1 & 0 & $1-20$ & 811 & END & & & & & \\
\hline$C E-144$ & 1 & 0 & $1-20$ & 811 & END & & & & & \\
\hline$N D-143$ & 1 & 0 & $1-20$ & 811 & END & & & & & \\
\hline ND-145 & 1 & 0 & $1-20$ & 811 & END & & & & & \\
\hline$P M-147$ & 1 & 0 & $1-20$ & 811 & END & & & & & \\
\hline$N D-147$ & 1 & 0 & $1-20$ & 811 & END & & & & & \\
\hline$S M-147$ & 1 & 0 & $1-20$ & 811 & END & & & & & \\
\hline$S M-149$ & 1 & 0 & $1-20$ & 811 & END & & & & & \\
\hline$S M-1.50$ & 1 & 0 & $1-20$ & 811 & END & & & & & \\
\hline$S M-151$ & 1 & 0 & $1-20$ & 811 & END & & & & & \\
\hline $5 M-152$ & 1 & 0 & $1-20$ & 811 & END & & & & & \\
\hline GD-155 & 1 & 0 & $1-20$ & 811 & END & & & & & \\
\hline EU-153 & 1 & 0 & $1-20$ & 811 & END & & & & & \\
\hline EU-154 & 1 & 0 & $1-20$ & 811 & END & & & & & \\
\hline$E U-155$ & 1 & 0 & $1-20$ & 811 & END & & & & & \\
\hline ZIRCALLO & & 2 & 1620 & END & & & & & & \\
\hline $\mathrm{H2O}$ & & 3 & $D E N=.7$ & 331 & & 570 & END & & & \\
\hline
\end{tabular}




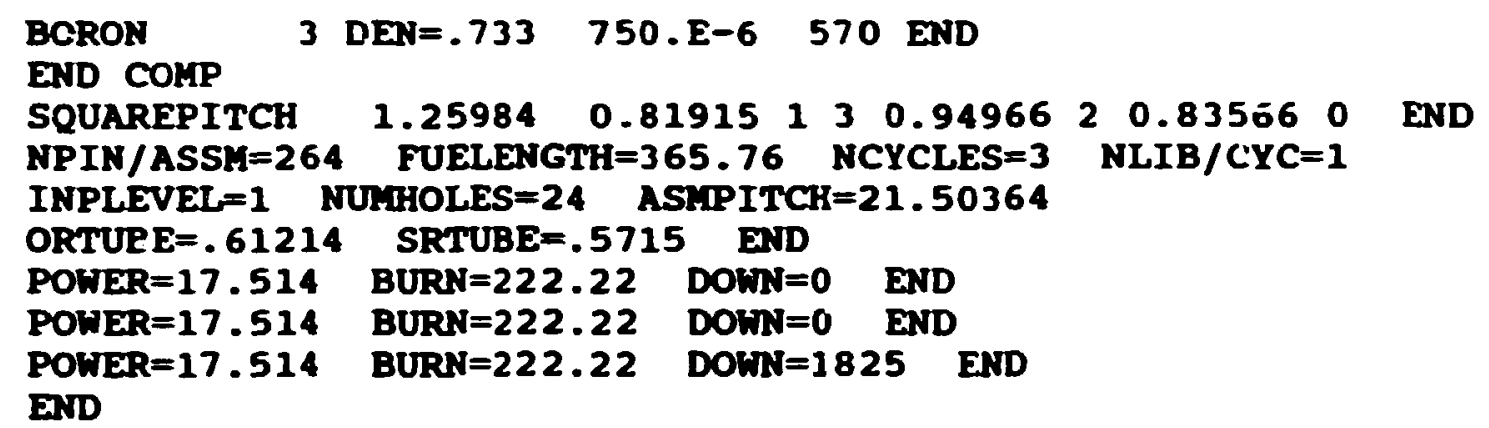


SAS2H INPUT DECK FOR $4.50 \mathrm{kT} 18000 \mathrm{MWD} / \mathrm{T}$ DEPLETION

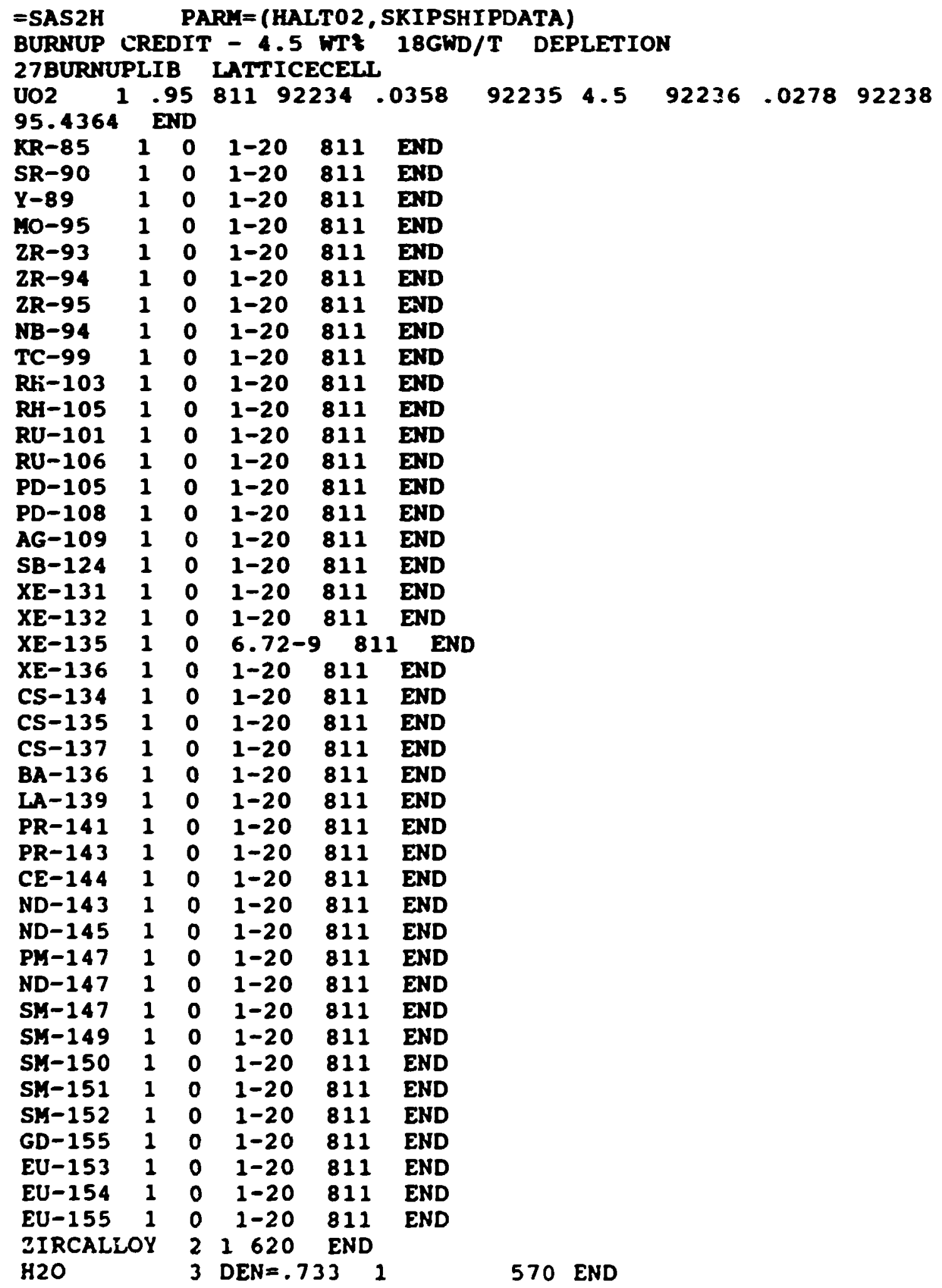




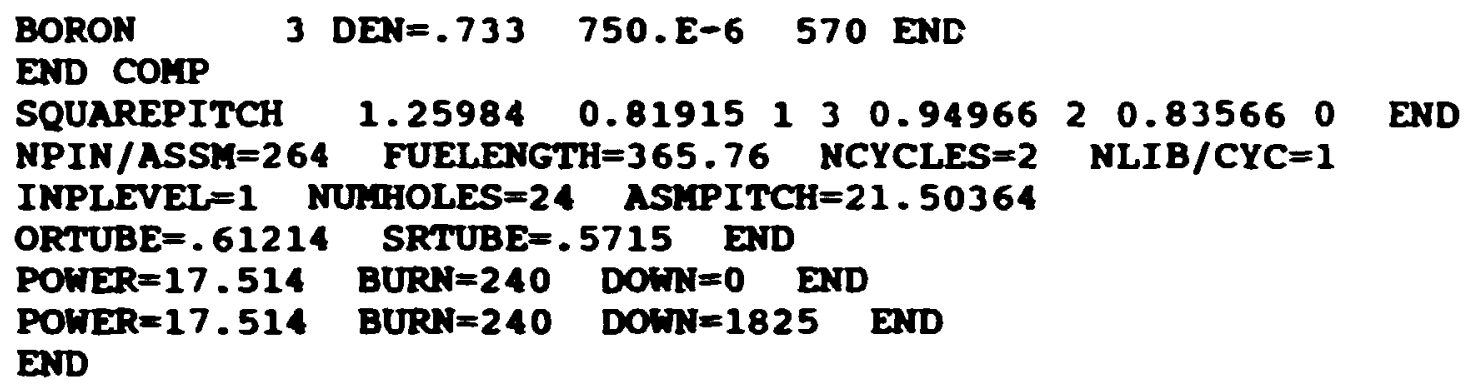


SAS2H INPUT DECK FOR 4.50 WT 25000 WWD/T DEPLETION

\begin{tabular}{|c|c|c|c|c|c|c|c|c|c|c|}
\hline $\begin{array}{l}=\text { SAS2H } \\
\text { BURNUP C } \\
27 \text { BURNUP }\end{array}$ & $\begin{array}{l}\text { RED } \\
\text { LIB }\end{array}$ & $\begin{array}{l}\mathbf{P A} \\
\mathbf{I T}\end{array}$ & $\begin{array}{l}R H=(H A \\
-4.5 \\
\text { LATTIC }\end{array}$ & $\begin{array}{l}\text { LT03, } \\
\text { WTZ } \\
\text { ECELL }\end{array}$ & $\begin{array}{l}\text { SKIPSH } \\
\text { 25GWD }\end{array}$ & $\begin{array}{l}\text { LPDATA } \\
\text { /T DE }\end{array}$ & PLETI & & & \\
\hline $\begin{array}{l}\text { UO2 } 1 \\
95.4364^{1}\end{array}$ & .9 & & 81192 & 234. & .0358 & 92235 & 4.5 & 92236 & .0278 & 92238 \\
\hline $\mathrm{KR}-85$ & 1 & 0 & $1-20$ & 811 & END & & & & & \\
\hline SR-90 & 1 & o & $1-20$ & 811 & END & & & & & \\
\hline Y-89 & 1 & $\mathbf{0}$ & $1-20$ & 811 & END & & & & & \\
\hline $180-95$ & 1 & 0 & $1-20$ & 811 & END & & & & & \\
\hline 2R-93 & 1 & 0 & $1-20$ & 811 & END & & & & & \\
\hline ZR-94 & 1 & 0 & $1-20$ & 811 & END & & & & & \\
\hline 2R-95 & 1 & 0 & $1-20$ & 811 & END & & & & & \\
\hline NB-94 & 1 & $\mathbf{0}$ & $1-20$ & 811 & END & & & & & \\
\hline TC-99 & 1 & 0 & $1-20$ & 811 & END & & & & & \\
\hline RH-103 & 1 & 0 & $1-20$ & $81 i$ & END & & & & & \\
\hline RH-105 & 1 & 0 & $1-20$ & 811 & END & & & & & \\
\hline$R U-101$ & 1 & 0 & $1-20$ & 811 & END & & & & & \\
\hline$R U-106$ & 1 & 0 & $1-20$ & 811 & END & & & & & \\
\hline$P D-105$ & 1 & 0 & $1-20$ & 811 & END & & & & & \\
\hline PD-108 & 1 & 0 & $1-20$ & 811 & END & & & & & \\
\hline AG-109 & 1 & 0 & $1-20$ & 811 & END & & & & & \\
\hline$S B-124$ & 1 & 0 & $1-20$ & 811 & END & & & & & \\
\hline$X E-131$ & 1 & 0 & $1-20$ & 811 & END & & & & & \\
\hline$X E-132$ & 1 & o & $1-20$ & 811 & END & & & & & \\
\hline$X E-135$ & 1 & o & $6.72-5$ & 981 & 11 END & & & & & \\
\hline$X E-136$ & 1 & 0 & $1-20$ & 811 & END & & & & & \\
\hline cs -134 & 1 & 0 & $1-20$ & 811 & END & & & & & \\
\hline Cs-135 & 1 & 0 & $1-20$ & 811 & END & & & & & \\
\hline Cs-137 & 1 & 0 & $1-20$ & 811 & END & & & & & \\
\hline$B A-136$ & 1 & 0 & $1-20$ & 811 & END & & & & & \\
\hline LA-139 & 1 & 0 & $1-20$ & 811 & END & & & & & \\
\hline PR-141 & 1 & $\mathbf{0}$ & $1-20$ & 811 & END & & & & & \\
\hline$P R-143$ & 1 & 0 & $1-20$ & 811 & END & & & & & \\
\hline$C E-144$ & 1 & $\mathbf{0}$ & $1-20$ & 811 & END & & & & & \\
\hline$N D-143$ & 1 & 0 & $1-20$ & 811 & END & & & & & \\
\hline ND-145 & 1 & 0 & $1-20$ & 811 & END & & & & & \\
\hline$P M-147$ & 1 & 0 & $1-20$ & 811 & END & & & & & \\
\hline ND-147 & 1 & 0 & $1-20$ & 811 & END & & & & & \\
\hline $5 M-147$ & 1 & 0 & $1-20$ & 811 & END & & & & & \\
\hline$S M-149$ & 1 & 0 & $1-20$ & 811 & END & & & & & \\
\hline$S M-150$ & 1 & 0 & $1-20$ & 811 & END & & & & & \\
\hline SH-151 & 1 & 0 & $1-20$ & 811 & END & & & & & \\
\hline$S M-152$ & 1 & 0 & $1-20$ & 811 & END & & & & & \\
\hline GD-155 & 1 & 0 & $1-20$ & 811 & END & & & & & \\
\hline$E U-153$ & 1 & 0 & $1-20$ & 811 & END & & & & & \\
\hline EU-154 & 1 & 0 & $1-20$ & 811 & END & & & & & \\
\hline EU-155 & 1 & 0 & $1-20$ & 811 & END & & & & & \\
\hline ZIRCALLO & DY & 2 & 1620 & END & & & & & & \\
\hline $\mathrm{H} 2 \mathrm{O}$ & & 3 & $D E N=.7$ & 331 & & 570 & END & & & \\
\hline
\end{tabular}




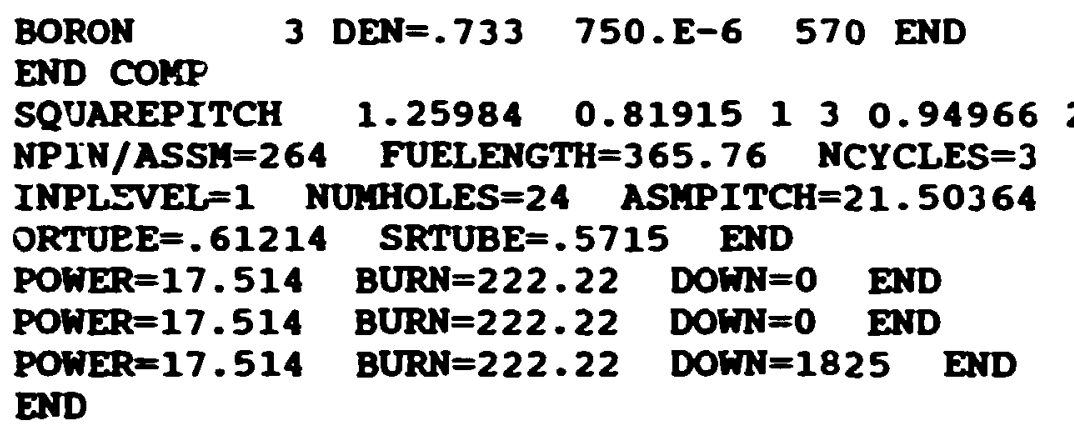


APPENDIX B 
SCALE INPUT DECK FOR 3AW-1484-7 EXPERIMENT 2282 (CORE IV) $=$ CSAS25

- PROBLEM ID BAW 1484-7 EXP 2282 (CORE IV)

' 2.468 U-235 ENRICHED UO2 RODS, 1.206 CM DIAM. AL CLAD, 1.044 CM ID,

- $1.030 \mathrm{CH}$ OD. UO2 $91.44 \mathrm{CH}$ LONG, H2O MODERATEC AND REFLECTED.

- CRITICAL H2O HEIGHT = $145.69 \mathrm{CM}$

- $914 \times 14$ CLUSTERS, SEPARATED BY 1 PIN PITCH WITH 84 B4C RODS

- REFERENCE: BAW-1484-7

- SCALE MOdULE: CSAS25

BAW 1484-7 CORE IV LATTICE PITCH=1.636 CM FUEL=?.? WT' ??000 MWD/T IN MOX

27BURNUPLIB LATM'ICECELL

$\checkmark U-23511005.67505-4$ END

U-238 1 1 0 2.22265-2 END

$101104.55881-2$ END

INSERT MIX 1 HERE

MG $2005.34873-4$ END

AL $\quad 205.80754-2$ END

SI $2004.63005-4$ END

CR $2001.09416-4$ END

MN $2004.43813-5$ END

FE $\quad 2002.03741-4$ END

CU $2001.02328-4$ END

H $300.6 .7755-2$ END

030 3.33877-2 END

B4C 4 DEN $=1.280 .9945$ END

$044003.32 .183-5$ END

MG $5005.34873-4$ END

AL $\quad 5 \quad 0 \quad 5.80754-2$ END

SI $\quad 5004.63000-4$ END

CR $5001.09416-4$ END

IN 5 5 5 4.43813-5 END

FE $5002.03741-4$ END

CU $501.02328-4$ END

H $\quad 6 \quad 0 \quad 6.67755-2$ END

$0 \quad 6 \quad 0 \quad 3.33877-2$ END

END COMP

SQUAREPITCH $1.6361 .030 \quad 131.20621 .044 \quad 0$ END

BAW-1484-7 CORE IV EXP 2282 ?.? WTళ ??000 MWD/ 9 ASSYS 14X14 W/ 84 B4C PINS

READ PARM PLT $=$ YES RUN $=Y E S$ GEN $=205$ NPG $=600$ NSK $=5$ TME $=30$

WRS $=34$ RES $=205$ END PARM

READ GEOM

UNIT 1

COM='BOTTOM PLUG OF FUEL ROD' 


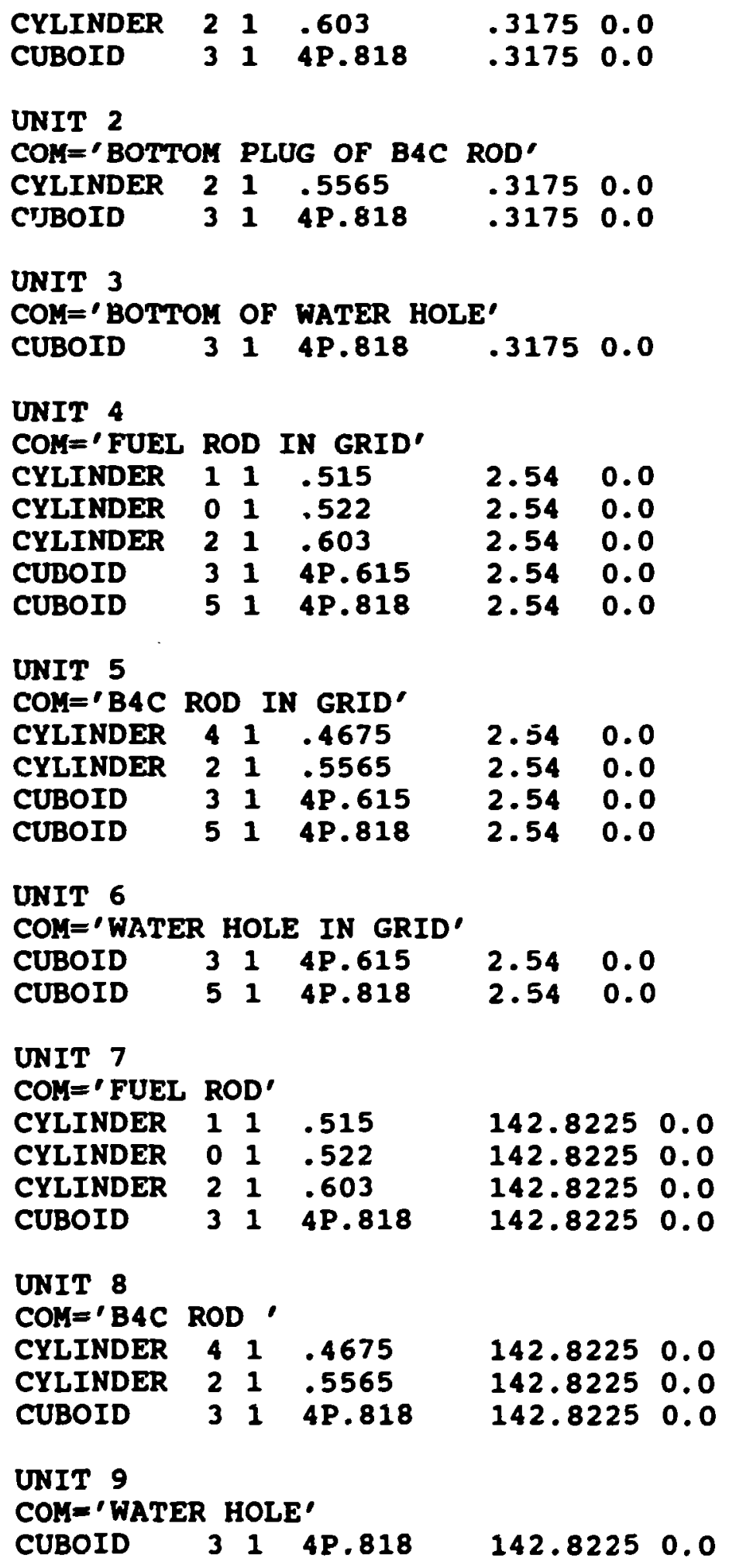

UNIT 11 


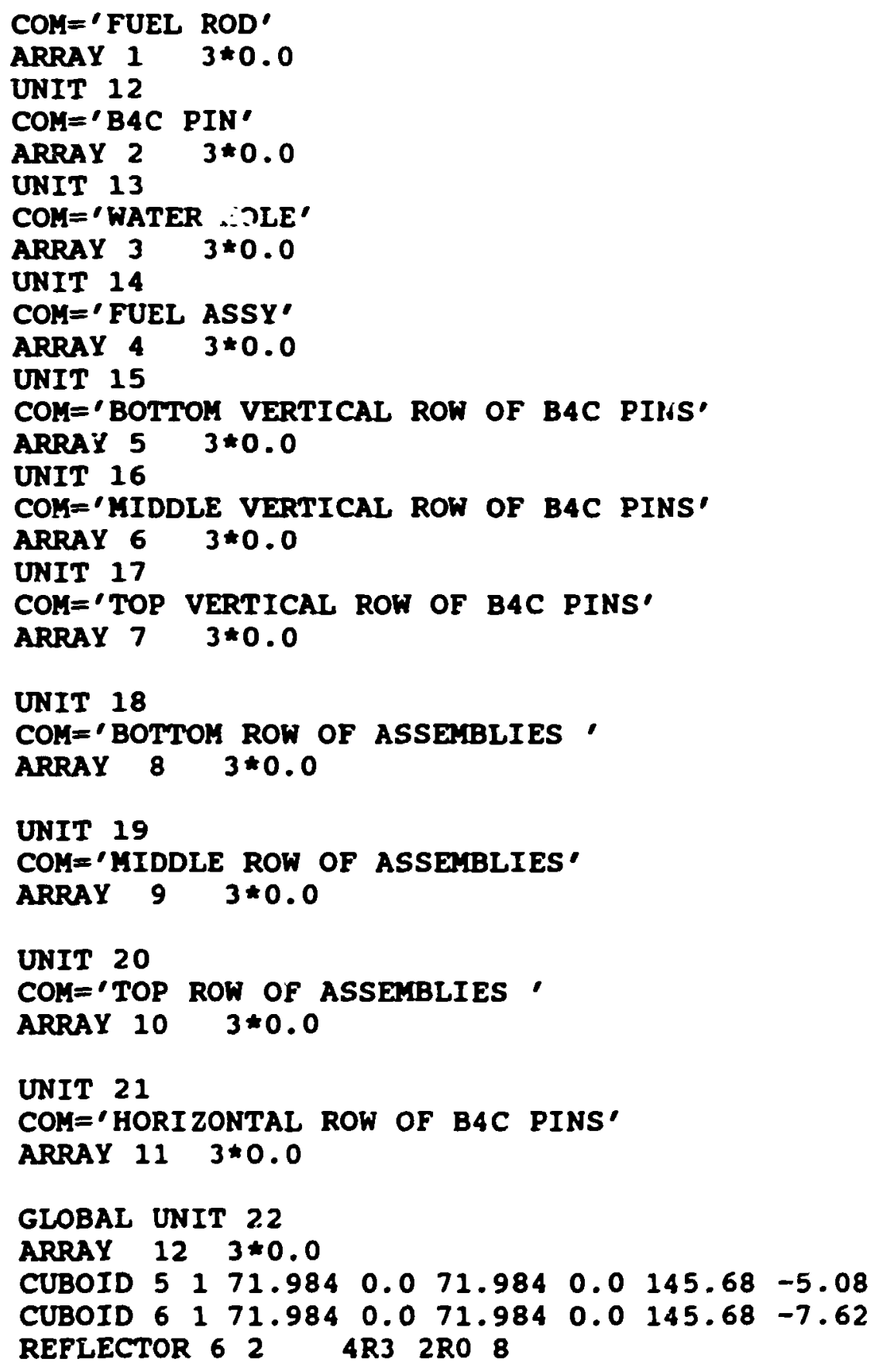


END FILL

COM='WATER HOLE' ARA=3 NUX=1 NUY=1 NUZ=3 FILL 369

END FILL

COM='FUEL ASSY' ARA=4 NUX=14 NUY=14 NUZ=1 FILL F11

END FILL

$A R A=5 \quad N U X=1 \quad N U Y=14 \quad N U Z=1$ FILL $1213 \quad 6 Q 2 \quad$ END FILL

$A R A=6$ NUX=1 NUY=14 NUZ=1 FILL $1312 \quad 2 Q 213 \quad 131213202$ END

FILL

$A R A=7$ NUX $=1$ NUY=14 NUZ=1 FILL 1312602 END FILL

$A R A=8$ NUX=5 NUY=1 NUZ=1 FILL $14 \quad 1514 \quad 1514$ END FILL

$A R A=9$ NUX=5 NUY=1 NUZ=1 FILL $141614 \quad 1614$ END FILL

$A R A=10$ NUX=5 NUY=1 NUZ=1 FILL 1417141714 END FILL

$A R A=11$ NUX $=44$ NUY $=1$ NUZ $=1$

FILI $13 \quad 13 \quad 12 \quad 1302 \quad 12 \quad 12 \quad 13 \quad 6 Q 2$ END FILL

ARA=12 NUX=1 NUY=5 NUZ=1 FILL $1821 \quad 192120$ END FILL

END ARRAY

READ PLOT

TTL=' $X-Y$ SLICE BELOW GRID PLATE'

$X U L=-0.5 \quad Y U L=72.5 \quad Z U L=0.2$

$X L R=72.5 \quad Y L R=-C .5 \quad Z L R=0.2$

$\mathrm{UAX}=1$ VDN $=-1$ NAX $=260$ NCH=' $12456789^{\circ}$ END

TTL $=$ ' $X-Y$ SLICE OF GRID PLATE'

$X U L=-0.5 \quad Y U L=72.5 \quad Z U L=2$.

$X L R=72.5 \quad Y L R=-0.5 \quad Z L R=2$.

UAX $=1$ VDN $=-1$ NAX $=260 \quad \mathrm{NCH}=0^{\prime} 12456789^{\prime}$ END

TTL $=$ ' $X-Y$ SLICE ABOVE GRID PLATE'

$X U L=-0.5 \quad Y U L=72.5 \quad Z U L=50$.

$X L R=72.5 \quad Y L R=-0.5 \quad Z L R=50$.

$U A X=1$ VDN $=-1$ NAX $=260 \quad \mathrm{NCH}=\prime 12456789^{\prime}$ END

END PLRT

END DATA

END 
SCALE INPUT DECK FOR PNL-7167 FLUX TRAP EXPERIMENT 214R

=CSAS2X

- 952 FUEL RODS

- 4.31 U-235 ENRICHED UO2 RODS, 1.265 CM DIAM.; AL CLAD, 1.283 CY ID,

1.415 CM OD. UO2 91.44 CM LONG, ROD 96.52 CM LONG. H2O MODERATED AND

- REFLECTED, 2 X 2 ARRAY OF FUEL ClUSters, 1.891 Cy SQUARE PITCH.

- 0.295 CY BETWEEN CLUSTERS AND POISON PLATES, 3.73 CM FLUX TRAP WIDTH.

- REFERENCE: PNL-7167

- scale module: csas2X

1.891 CM PITCH LATTICE CELL, ?.? WT ENRICHED FUEL, ??000 MUD/T

27BURNUPLIB LATTICECELL

$\begin{array}{llllllllllll} & \text { UO2 } & 1 & 0.9489 & 293 & 92234 & 0.022 & 92235 & 4.306 & 92236 & 0.022 & 92238\end{array}$ 95.650 END

INSERT MIX 1 HERE

AL 2 END

H2O 3 END

$\begin{array}{llllllllllll}\text { 'UO2 } & 8 & 0.9489 & 293 & 92234 & 0.022 & 92235 & 4 . j 06 & 92236 & 0.022 & 52238\end{array}$

95.650 END

INSERT MIX 8 HERE

AL 9 END

H2O 10 END

AL 4 END

$\begin{array}{llllllllllll}A R B M B O R A L & 2.64 & 5 & 1 & 0 & 0 & 5000 & 29.22 & 6012 & 8.16 & 13027 & 62.54\end{array}$

$$
80160.06260000 .025 \text { END }
$$

ARBMRUBBER $1.321 \quad 6 \quad 0 \quad 0 \quad 0 \quad 6012 \quad 58.1001 \quad 6.5 \quad 20000 \quad 11.4$

H2O 7 END

END COMP

SQUAREPITCH $1.891 \quad 1.265 \quad 1 \quad 3 \quad 1.41521 .283 \quad 0$ END

MORE DATA

RES $=8$ CYLINDER .6325 DAN $(8)=.173084$

END MORE

FLUX TRAP ASSEMBLY NO. $214 \mathrm{R}$ W/ ?.? WT' UO2 ??000 MWD/T

READ PARY PLT $=Y E S$ RUN=YES GEN $=205$ NPG $=600$ NSK=5 TME=30 WRS $=34$ RES $=205$ END PARM

READ GEOMETRY

UNIT 1

CYLINDER $810.6325 \quad 2$ P45.72

CYLINDER $0 \quad 1 \quad .6415$ 2P45.72

CYLINDER $6 \quad 1.6415 \quad 2 P 48.26$

CYLINDER $91.7075 \quad 2 P 48.26$

CUBOID $10 \quad 1 \quad 4$ P.9. $5 \quad 2$ P48.26

UNIT 2

ARRAY $13 * 0.0$ 


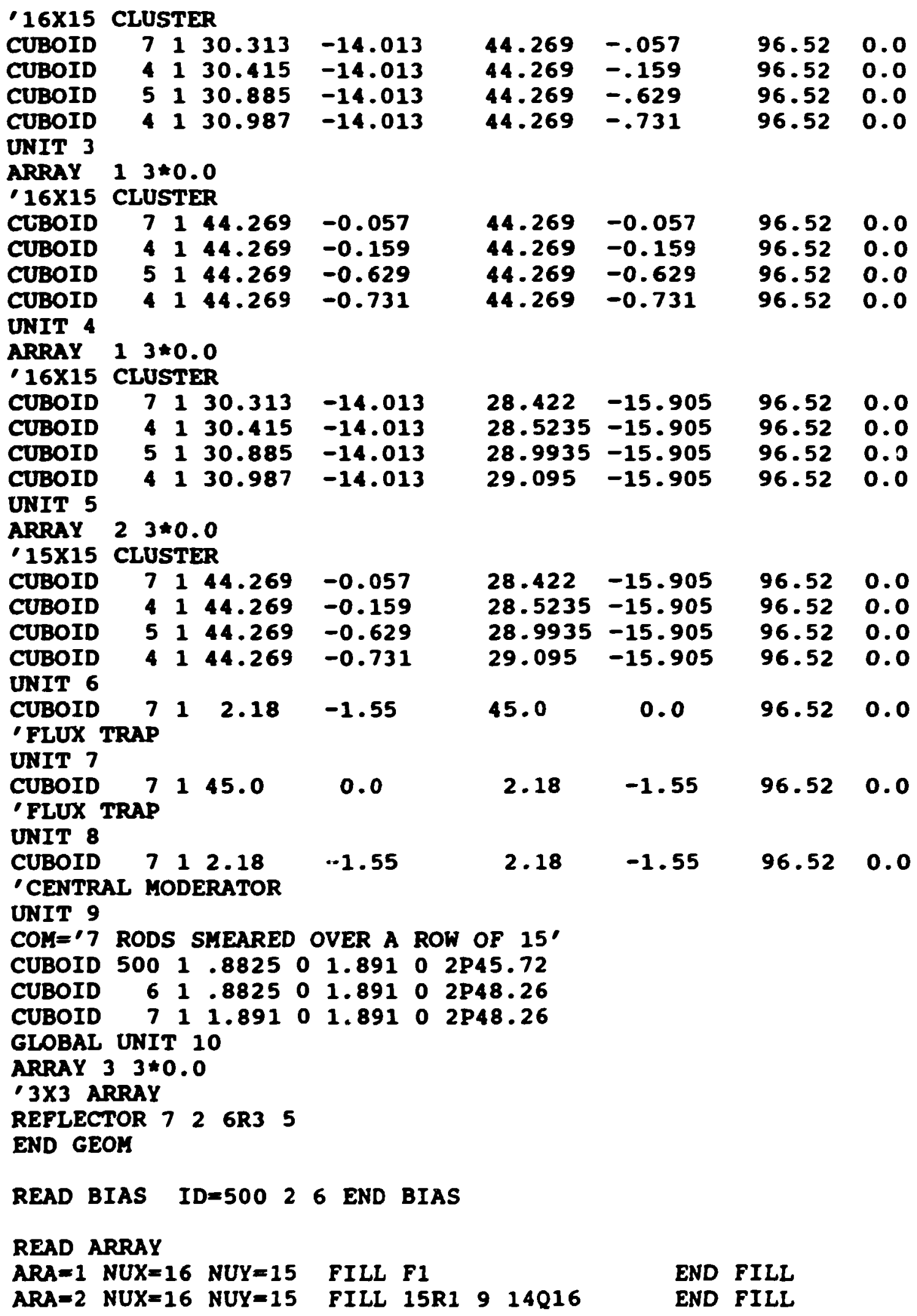




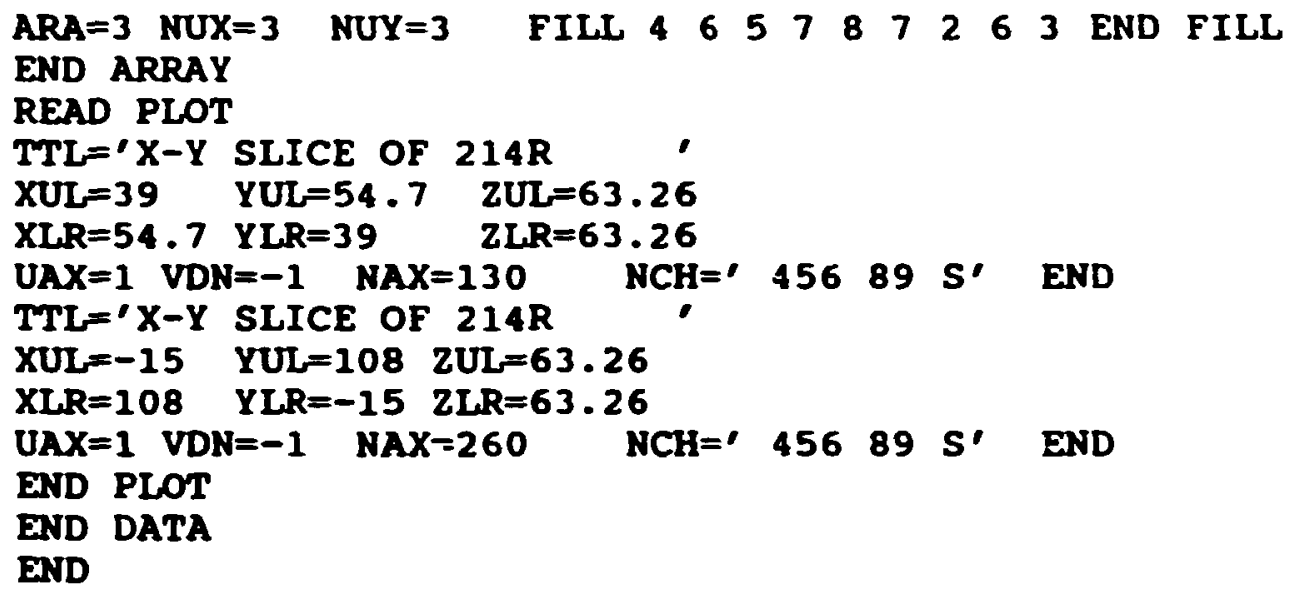


SCALE INPUT DECK FOR PNL-7167 FLUX TRAP EXPERIMENT $214 \mathrm{V3}$

$=\operatorname{CSAS} 2 \mathrm{X}$

- 862.1 FUEL RODS

- 4.31 U-235 ENRICHED UO2 RODS, 1.265 CH DIAM.; AL CLAD, 1.283 CH ID,

- 1.415 CI OD. UO2 91.44 CI LONG, ROD 96.52 CM LONG. H2O MODERATED AND

- REFLECTED, 2 X 2 ARRAY OF FUEL CLUSTERS, 1.891 CA SQUARE PITCH.

- 0.295 CA BETweEN CLUSTERs AND POISON PLATES, 3.73 CA FLUX TRAP WIDTH.

- three 0.63 CH X 45.0 CH X 96 ch al pLATES between clusters.

- REFERENCE: PNL-7167

- scale hodULE: CSAS2X

-

1.891 CH PITCH LATTICE CELL, ?.? WT ENRICHED ??000 MWD/T 27BURNUPLIB LATTICECELL

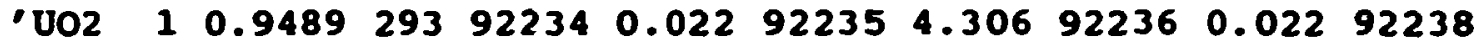
95.650 END

INSERT MIX 1 HERE

AL 2 END

H2O 3 END

$\begin{array}{llllllllllll} & \text { UO2 } & 8 & 0.9489 & 293 & 92234 & 0.022 & 92235 & 4.306 & 92236 & 0.022 & 92238\end{array}$

95.650 END

INSERT MIX 8 HERE

AL 9 END

H2O 10 END

AL 4 END

$\begin{array}{lllllllllll}A R B M B R A L & 2.645 & 1 & 0 & 0 & 5000 & 29.22 & 6012 & 8.16 & 13027 & 62.54\end{array}$ 80160.06260000 .025 END

ARBMRUBBER 1.32160000601258 .10016 .52000011 .4

H2O 7 END

END COMP

SQUAREPITCH 1.8911 .265131 .41521 .2830 END

MORE DATA

RES $=8$ CYLINDER .6325 DAN $(8)=.173084$

END MORE

FLUX TRAP ASSEABLY NO. $214 \mathrm{V3} ?$ ? ? WTt ??000 MWD/T

READ PARY PLT=YES RUN=YES GEN=205 NPG=600 NSK=5 TME=30

READ GEOMETRY

WRS $=34$ RES $=205$ END PARM

UNIT 1

CYLINDER $81.6325 \quad 2$ P45.72

CYLINDER $011.6415 \quad 2 P 45.72$

CYLINDER $6 \quad 1 \quad .6415 \quad 2 P 48.26$

CYLINDER $91.7075 \quad 2 P 48.26$

CUBOID $10 \quad 1 \quad 4$ P.9455 2 P48.26

UNIT 2

ARRAY $13 * 0.0$ 


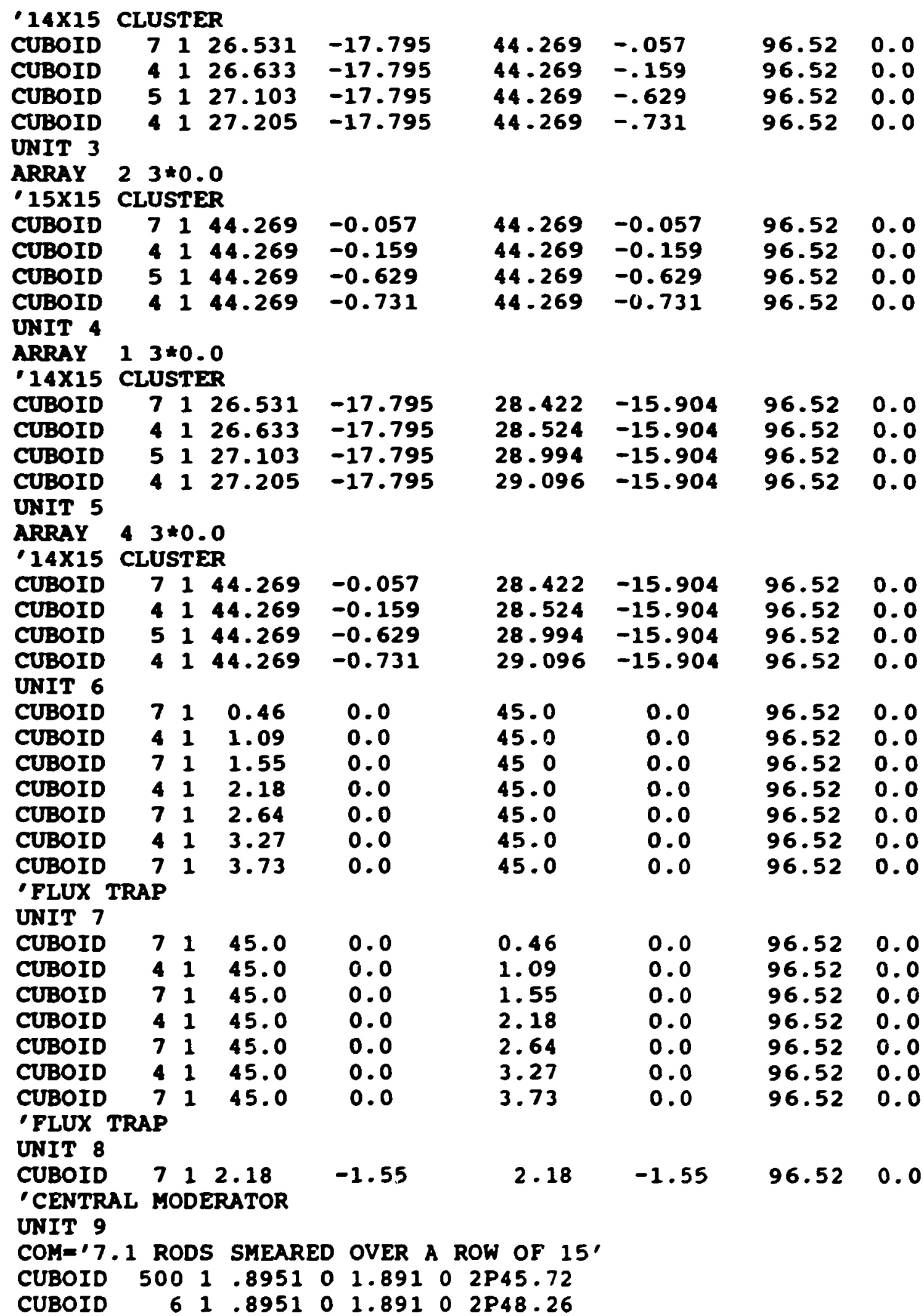




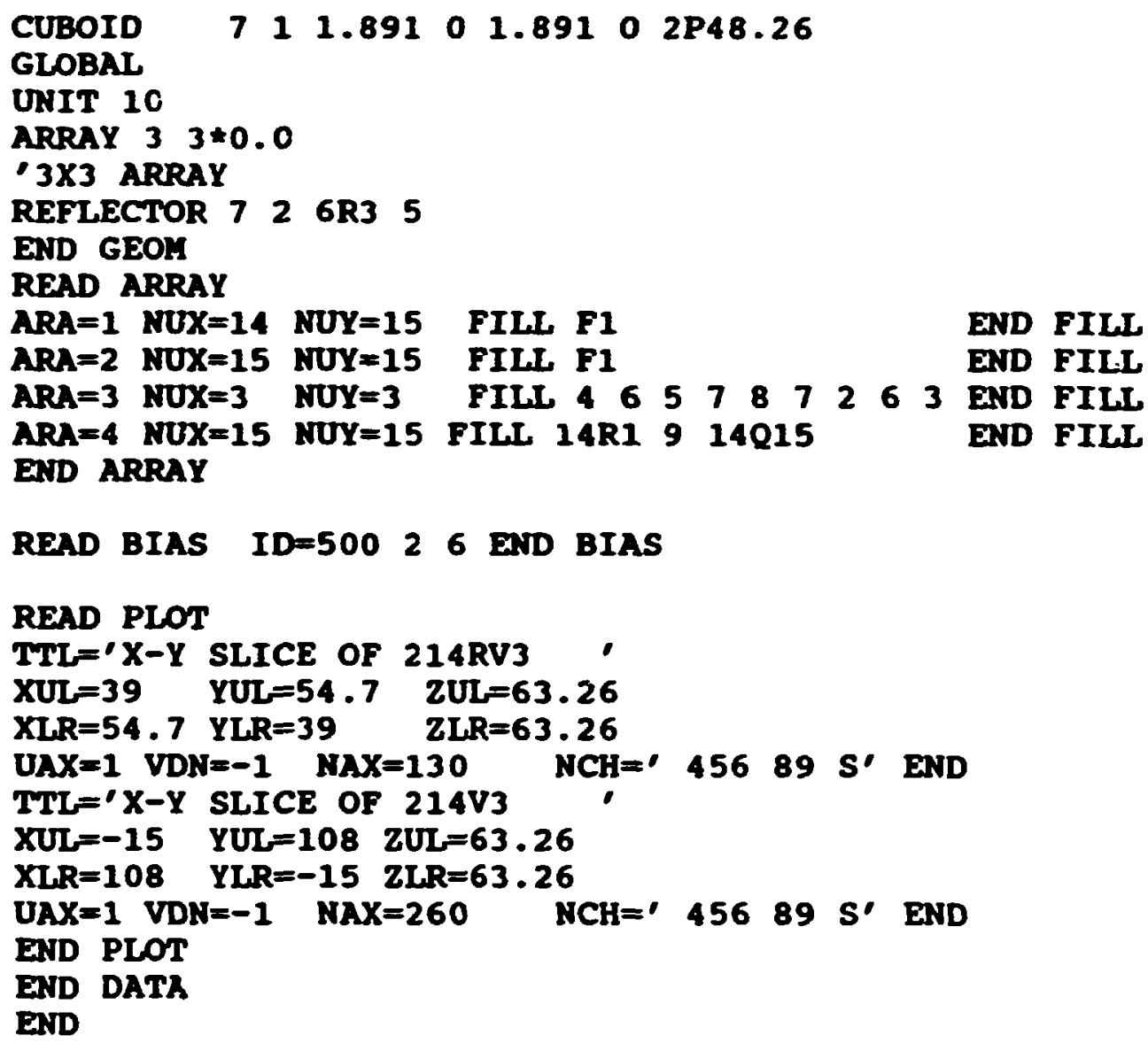


SCALE INPUT DECK FOR PNL-3602 CASE

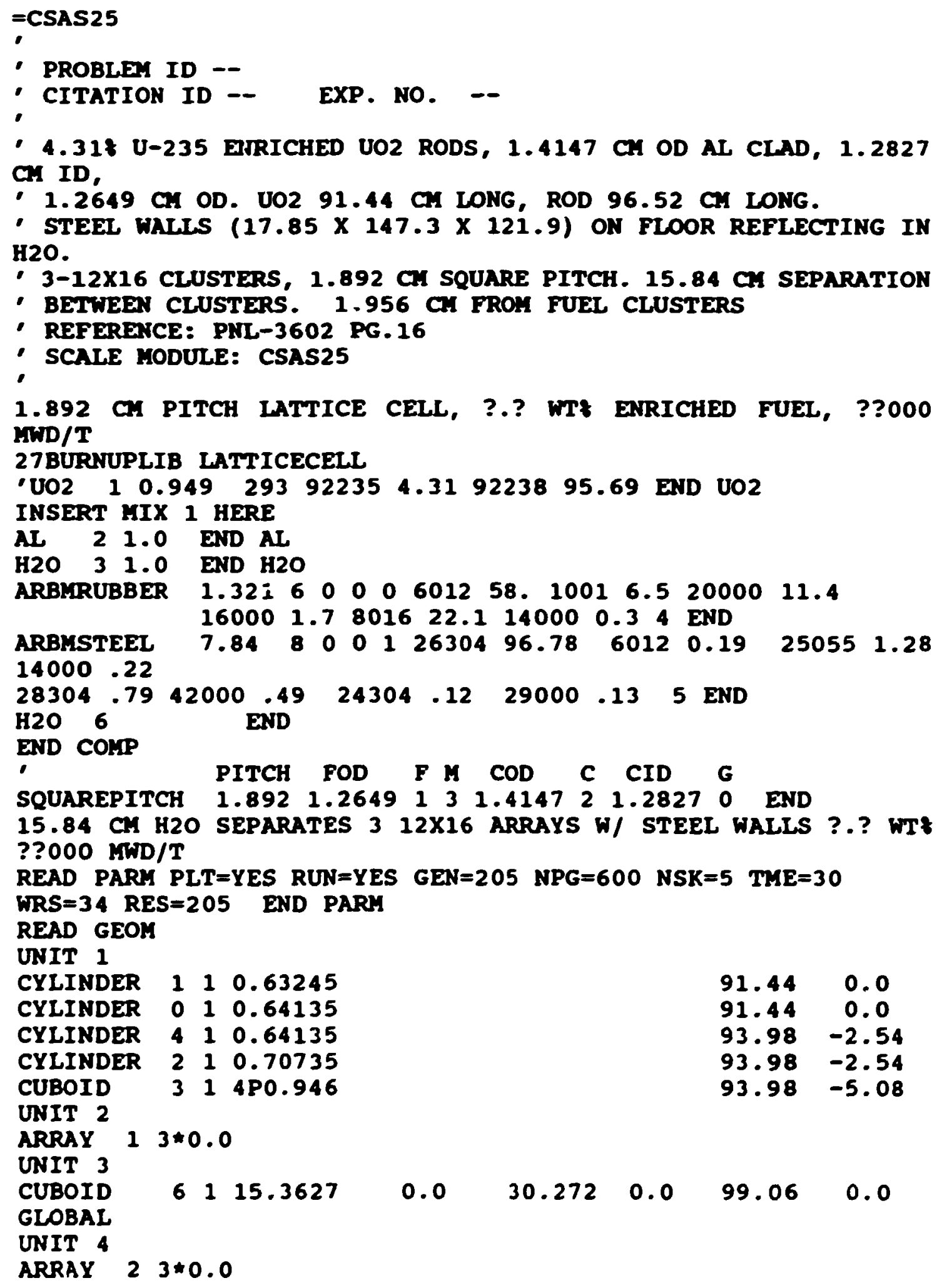

1.892 CH PITCH LATTICE CELL, ?.? WT' ENRICHED FUEL, ??000 MWD/T

$$
\begin{array}{ll}
91.44 & 0.0 \\
91.44 & 0.0 \\
93.98 & -2.54 \\
93.98 & -2.54 \\
93.98 & -5.08
\end{array}
$$




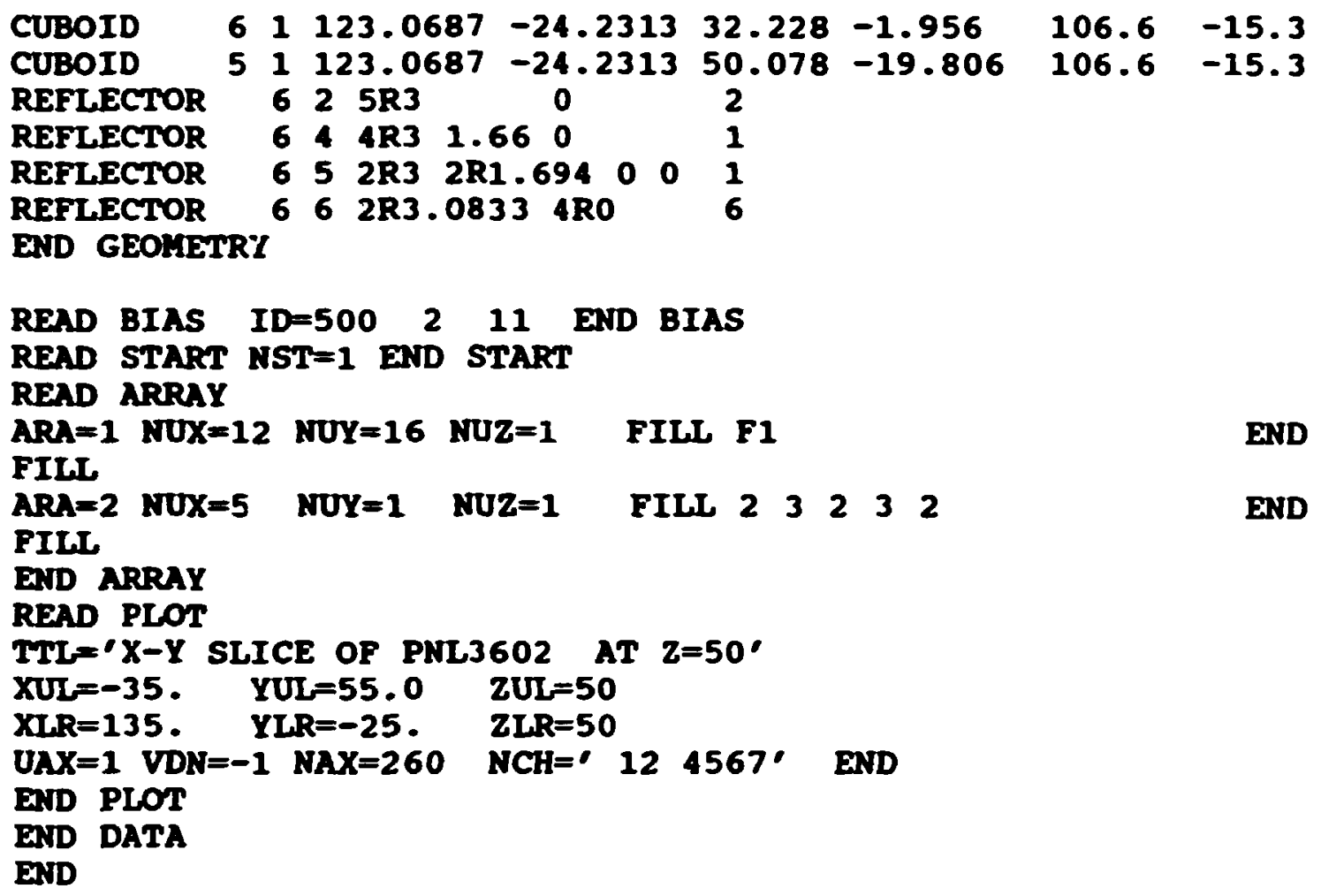


SCALE INPUT DECK FOR PNL-3926 DEPLETED URANIUM WALLS CASE

$=\operatorname{CSAS25}$

- PROBLEA ID --

- CITATION ID -- EXP. NO. --

- 4.317 U-235 ENRICHED UO2 RODS, 1.415 CM OD AL CLAD, 1.2827 CAI ID,

- 1.2649 CH OD UO2 91.44 CH LONG, ROD 96.52 CH LONG.

- URANIUA WALLS (7.65 X $152.3 \times 121.9)$ ON FLOOR REFLECTING IN H2O.

- 3-12X16 CLUSTERS, 1.892 CH SQUARE PITCH. 19.24 CH SEPARATION

- BETWEEN CLUSTERS. , 1.956 CH FROH FUEL CLUSTERS

- REFERENCE: PNL-3926 PG.13

- scale module: csas25

-

1.892 IN PITCH LATTICE CELL, ?.? WT: ENRICHED FUEL, ??000 MWD/MTU

27BURNUPLIB LAT I ICECELL

INSERT MIX 1 HERE

AL 21.0 END AL

H2O 31.0 END H2O

ARBIRUBBER $1.32160000601258 .10016 .5 \quad 2000011.4$

$160001.7801622 .114000 \quad 0.34$ END

$\begin{array}{lllllllll}\text { URANIUM } & 5 & 0.9816 & 293 & 92235 & 0.199 & 92238 & 99.801 & \text { END }\end{array}$

H2O 6 END

END COMP

- PITCH FOD F M COD C CID G

SQUAREPITCH $1.892 \quad 1.2649 \quad 1 \quad 3 \quad 1.4147 \quad 2 \quad 1.2827 \quad 0 \quad$ END

PNL-3926 DEPL. U REFLECTING WALLS, ?.? WT' UO2 ??000 MWD/MTU

READ PARM PLT=YES RUN=YES GEN $=205$ NPG $=600$ NSK $=5$ TME $=30$

WRS $=34$ RES $=205$ END PARM

READ GEOM

UNIT 1

CYLINDER 1110.63245

CYLINDER $0 \quad 1 \quad 0.64135$

CYLINDER $4 \quad 1 \quad 0.64135$

CYLINDER 2 2 10.70735

CUBOID $\quad 314$ P0.946

91.440 .0

91.440 .0

$93.98-2.54$

$93.98-2.54$

UNIT 2

$93.98-5.08$

ARRAY $13 \star 0.0$

UNIT 3

CUBOID

GLOBAL

UNIT 4

ARRAY $23 * 0.0$

$\begin{array}{llllllllllll}\text { CUBOID } & 6 & 1 & 128.9687 & -23.3313 & 32.228 & -1.956 & 106.6 & -15.3\end{array}$

$\begin{array}{llllllllllll}\text { CUBOID } & 5 & 1 & 128.9687 & -23.3313 & 39.878 & -9.606 & 106.6 & -15.3\end{array}$

REFLECTOR 622 5R3 022

REFLECTOR $6 \begin{array}{llllllll}6 & 4 & 4 & 1.66 & 0 & 1\end{array}$ 
REFLECTOR $6 \quad 5 \quad 4 R 3 \quad 2 R O \quad 3$

REFLECTOR 6 8 2 2R3 2R2.894 2RO 1

REFLECTOR $6 \quad 9 \quad 2 R 3.167 \quad 4 R O \quad 3$

END GEOMETRY

READ BIAS ID $=500211$ END BIAS

READ START NST=1 END START

READ ARRAY

$A R A=1$ NUX $=12$ NUY $=16$ NUZ=1 FILL F1 END

FILL

$A R A=2$ NUX $=5 \quad N U Y=1 \quad N U Z=1 \quad$ FILL $\begin{array}{lllll}2 & 3 & 2 & 3 & 2\end{array}$

END

FILL

END ARRAY

READ PLOT

TTL=' $X-Y$ SLICE OF PNL3926A AT $Z=50^{\prime}$

$X U L=-27$. YUL $=45.0 \quad Z U L=50$

$X L R=135 . \quad Y L R=-15 \quad 2 L R=50$

$U A X=1$ VDN $=-1$ NAX $=260$ NCH $=\prime: 24567^{\prime}$ END

END PLOT

END DATA

END 
SCALE INPUT DECK FOR PNL-3926 LEAD WALLS CASE

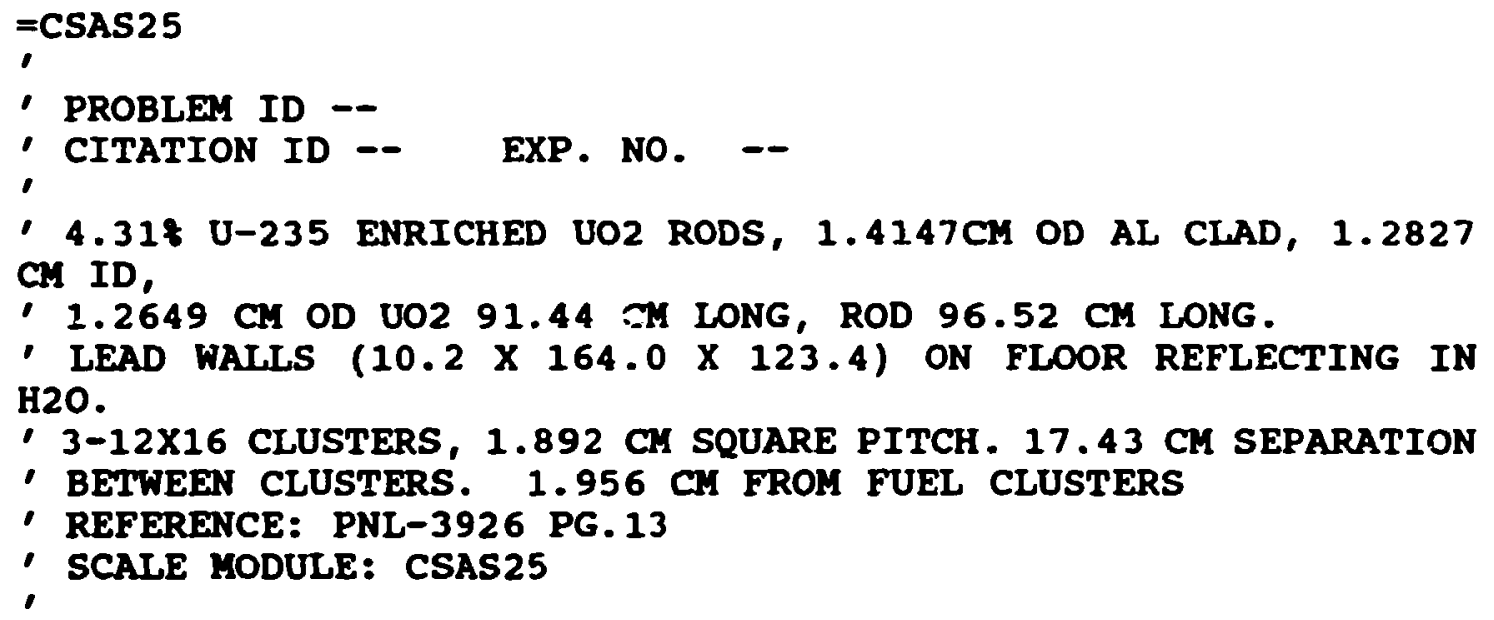

1.892 IN PITCH LATTICE CELL, ?.? WT\& ENRICHED FUEL, ??000 MWD/T

0

$\begin{array}{lc}91.44 & 0.0 \\ 9.1 .44 & 0.0 \\ 93.98 & -2.54 \\ 93.98 & -2.54 \\ 93.98 & -5.08\end{array}$




$\begin{array}{lrllllllll}\text { REFLECTOR } & 6 & 4 & 4 R 3 & 0.16 & 0 & & & 1 \\ \text { REFLECTOR } & 6 & 5 & 4 R 3 & 0 & 0 & & & 3 \\ \text { REFLECTOR } & 6 & 8 & \text { 2R3 } & 2 R .344 & 0 & 0 & 1 \\ \text { REFLECTOR } & 6 & 9 & \text { 2R3 } & 167 & 4 R 0 & & & 3 \\ \text { END GEOMETRY } & & & & & & & \end{array}$

READ BIAS ID $=5002$ 11 END BIAS

READ START NST=1 END START

READ ARRAY

ARA $=1$ NUX $=12$ NUY $=16$ NUZ=1 FILL F1 END

FILL

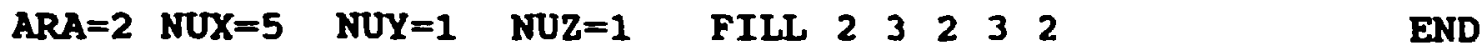

FILL

END ARRAY

READ PLOT

TTL $=' X-Y$ SLICE OF PNL3926B AT $Z=50^{\prime}$

$X U L=-35 . \quad Y U L=45.0 \quad Z U L=50$

$X L R=135$. YLR $=-15 . \quad Z L R=50$

$\mathrm{UAX}=1 \mathrm{VDN}=-1 \mathrm{NAX}=260 \mathrm{NCH}={ }^{\prime} 124567^{\prime}$ END

END PLOT

END DATA

END 
SCALE INPUT DECK FOR PNL-4257 EXPERIMENT 173

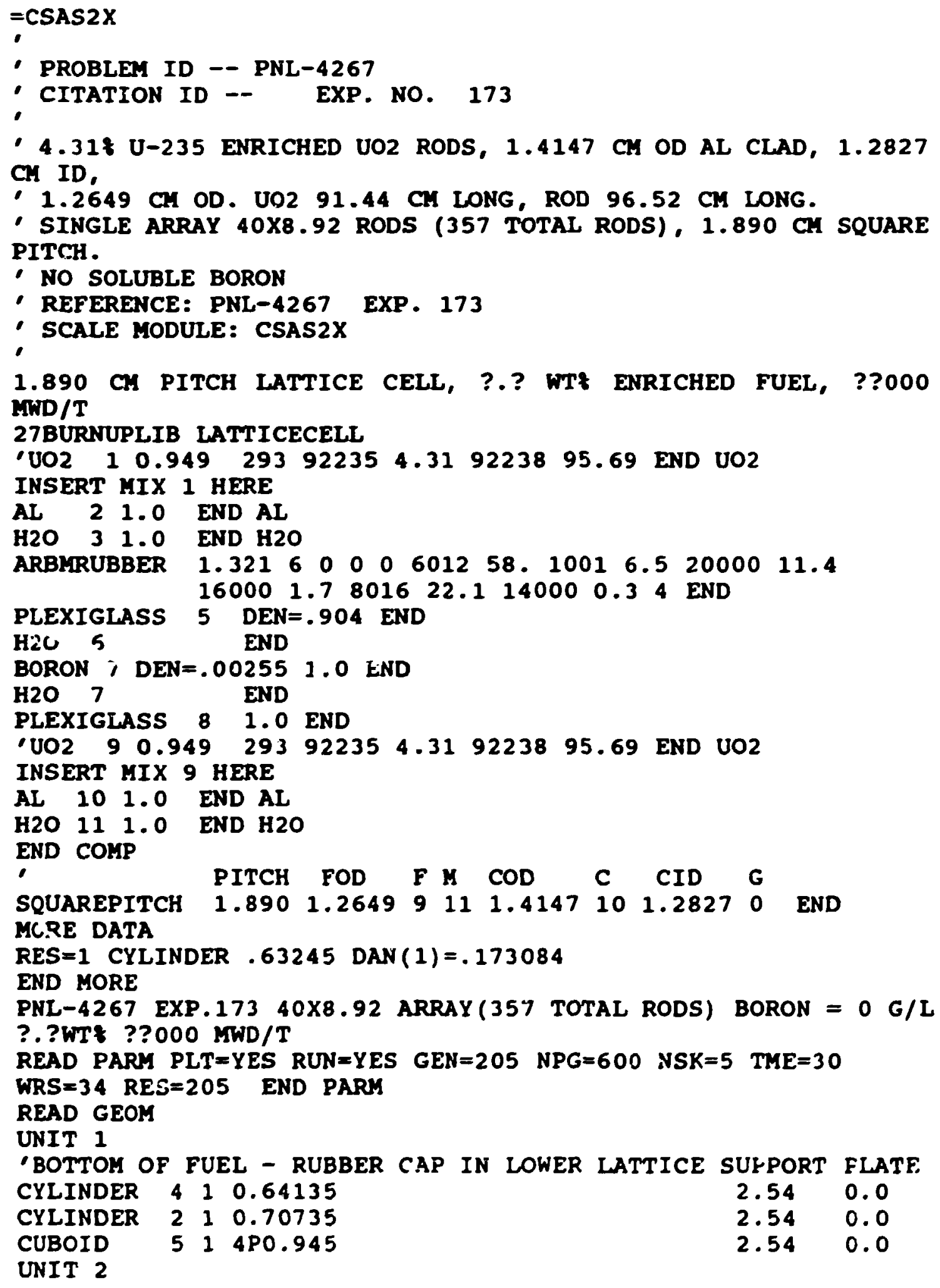


'FUEL BETWEEN THE TWO LATTICE SUPPORT PLATES CYLINDER 1110.63245

CYLINDER $\quad 0 \quad 1 \quad 0.64135$

$67.29 \quad 0.0$

CYLINDER 2110.70735

$67.29 \quad 0.0$

CUBOID $\quad 314$ P0.945

$67.29 \quad 0.0$

UNIT 3

' FUEL IN UPPER LATTICE SUPPORT PLATE

CYLINDER 1110.63245

CYLINDER 0010.64135

CYLINDER $2 \quad 1 \quad 0.70735$

CUBOID 514 P0.945

UNIT 4

- FUEL ABOVE THE UPPER LATTICE SUPPORT PLATE

CYLINDER 110.63245

CYLINDER 0010.64135

CYLINDER $2 \quad 1 \quad 0.70735$

CUBOID 314 P0.945

67.290 .0

UNIT 5

' BOTTOM OF SMEARED FUEL

CUBOID $500 \quad 1 \quad 1.7483 \quad 0 \quad 2 P 0.945$

CUBOID $\quad 3111.890 \quad 0 \quad 2$ P0.945

UNIT 6

- SMEARED FUEL BETWEEN THE TWO LATTICE SUPPORT PLATES

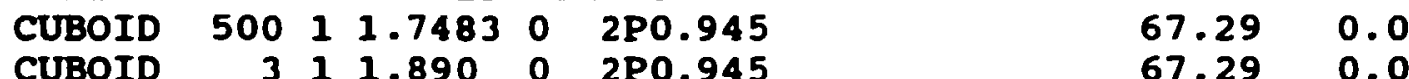

UNIT 7

'SMEARED FUEL IN UPPER LATTICE SUPPORT PLATE

$\begin{array}{llllllll}\text { CUBOID } 500 \quad 11.7483 & 0 & 2 P 0.945 & 1.27 & 0.0\end{array}$

CUBOID $\quad 3 \quad 11.890 \quad 0 \quad 2 P 0.945$

$1.27 \quad 0.0$

UNIT 8

'SMEARED FUEL ABOVE THE UPPER LATTICE SUPPORT PLATE

CUBOID 500111.7483002 P0.945

CUBOID $\quad 3 \quad 1 \quad 1.890 \quad 0 \quad 2 P 0.945$

$25.4 \quad 0.0$

GLOBAL

UNIT 9

ARRAY $1-8.505 \quad-37.80 \quad 0.0$

$\begin{array}{lllllll}\text { CUBOID } & 3 & 1 & 2 \mathrm{P} 39.80 & 2 \mathrm{P} 37.94 & 111.7 & 0.0\end{array}$

$\begin{array}{lllllll}\text { CUBOID } & 8 & 1 & 2 \mathrm{P} 41.705 & 2 \mathrm{P} 39.845 & 111.7 & -17.105\end{array}$

REFLECTOR $6 \quad 2 \quad$ 4R3 2RO 10

END GEOMETRY

READ BIAS ID $=500211$ END BIAS

READ ARRAY

$A R A=1$ NUX=9 NUY=40 NUZ=4 FILL 8R1 5 39Q9 $8 R 2 \quad 6 \quad 39 Q 9$

END FILL $\begin{array}{llllll}8 R 3 & 7 & 3909 & 8 R 4 & 8 & 3909\end{array}$

END ARRAY

READ PLOT

TTL $=' X-Y$ SLICE OF PNL4267A AT $2=50^{\prime}$

$X U L=-45 . \quad Y U L=45.0 \quad Z U L=50$ 
$X L R=45 . \quad Y L R=-45 . \quad Z L R=50$

UAX $=1$ VDN $=-1$ NAX $=130$ NCH $=\prime 124578 \&^{\prime}$ END

END PLOT

END DATA

END 
SCALE INPUT DECK FOR PNL-4267 EXPERIMENT 177

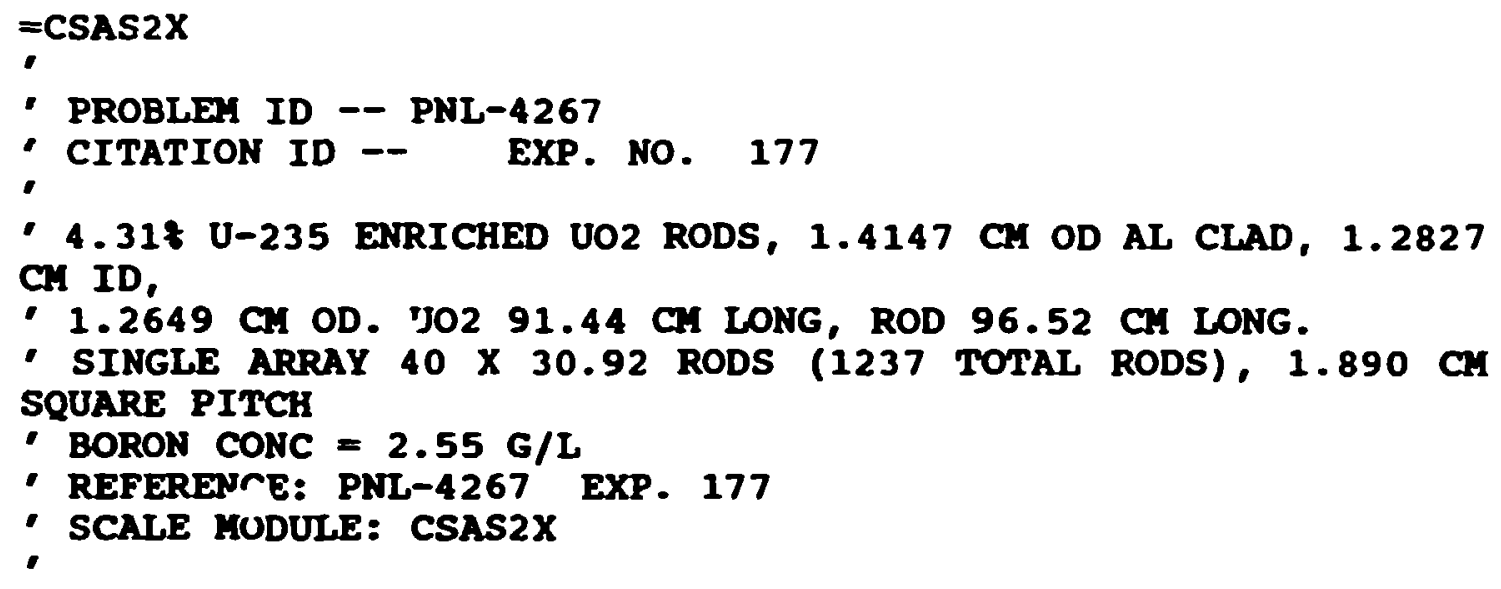

$\begin{array}{ll}2.54 & 0.0 \\ 2.54 & 0.0 \\ 2.54 & 0.0\end{array}$




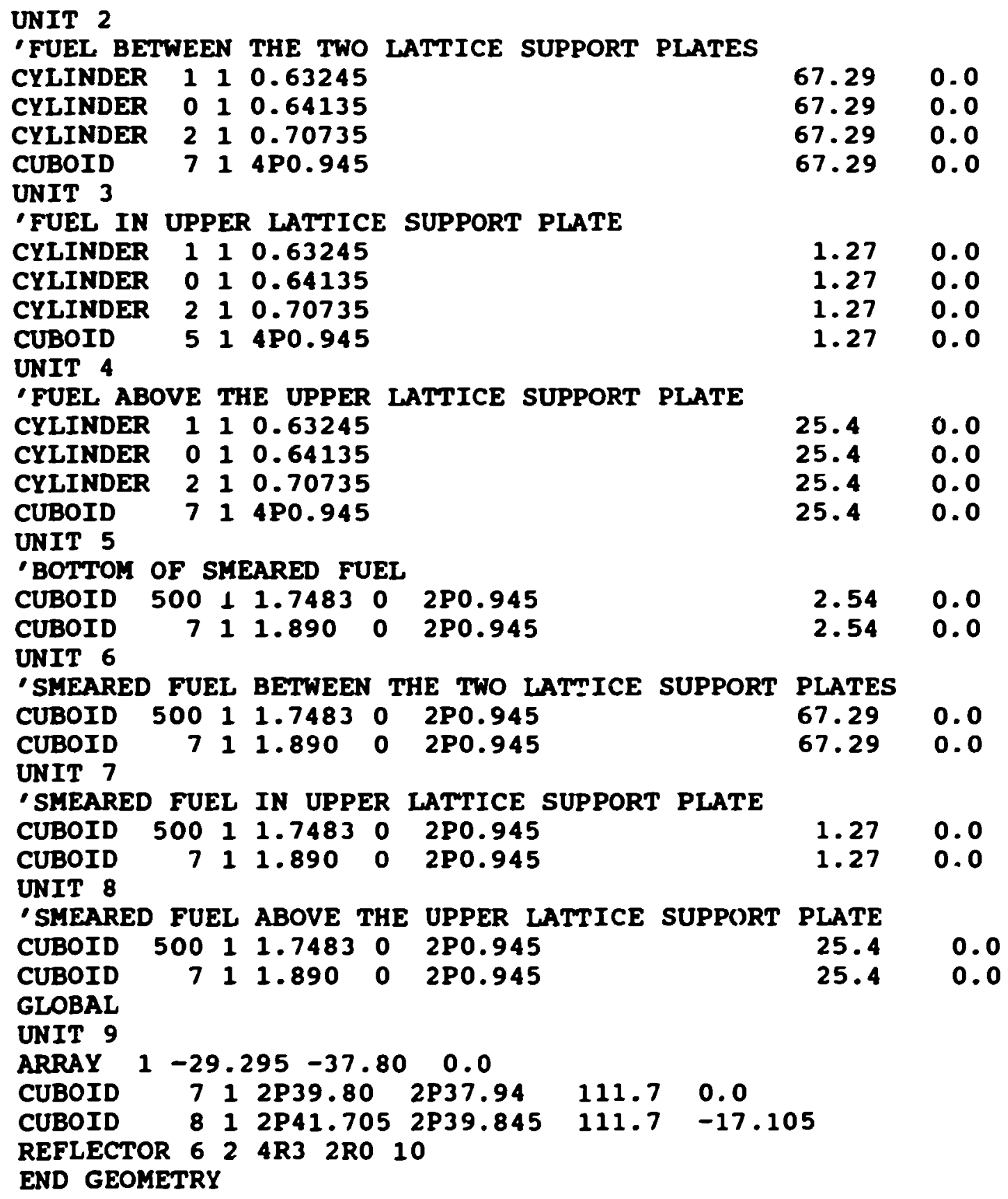

READ BIAS ID $=500211$ END BIAS

READ ARRAY

$A R A=1$ NUX $=31$ NUY $=40$ NUZ $=4$ FILL $30 R 15 \quad 39 Q 31 \quad 30 R 2 \quad 6 \quad 39 Q 31$ $\begin{array}{lllll}30 R 37 & 39031 & 30 R 4 & 8 & 39031\end{array}$

END FILL

END ARRAY

READ PLOT

TTL $=' X-Y$ SLICE OF PNL4267B AT $Z=50^{\prime}$ 
$X U L=-45 . \quad Y U L=45.0 \quad Z U L=50$

$X I R=45 . \quad Y L R=-45 . \quad Z L R=50$

$\mathrm{UAX}=1 \mathrm{VDN}=-1 \mathrm{NAX}=130 \mathrm{NCH}={ }^{\prime} 1245784^{\prime}$ END

END PLOT

END DATA

END 
SCALE INPUT DECK FOR PNL-4267 EXPERIMENT 178

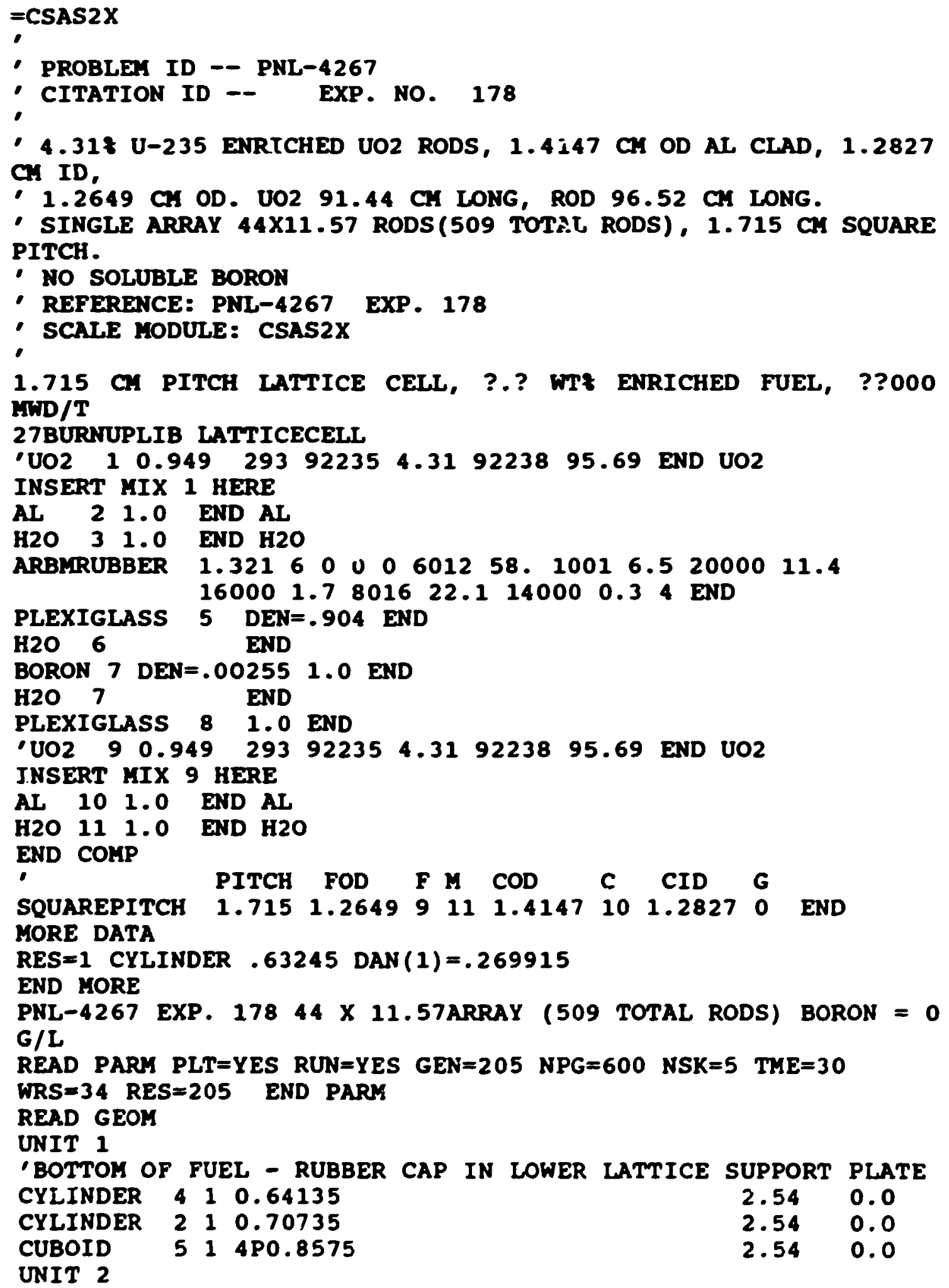




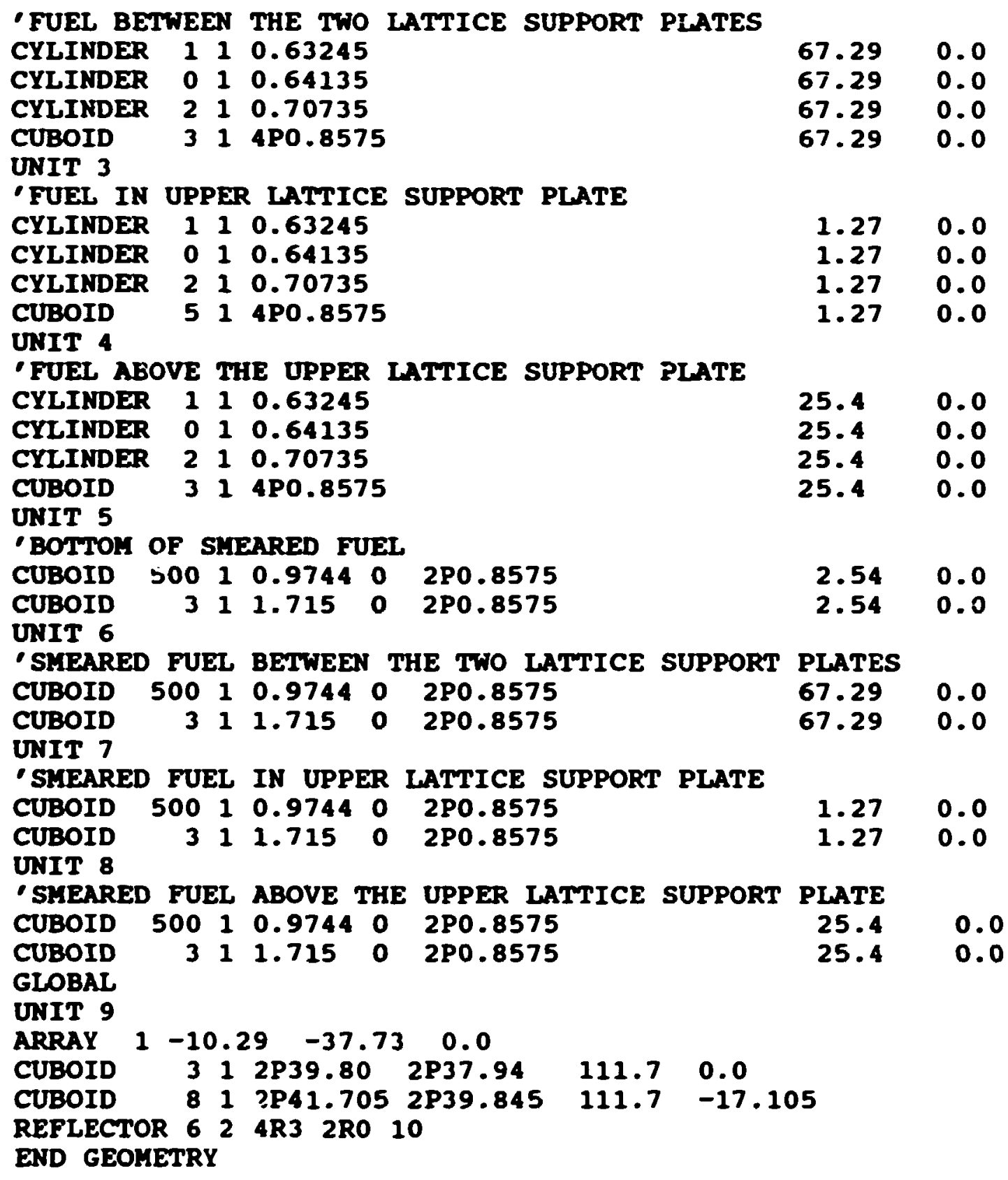

READ BIAS ID $=500211$ END BIAS

READ ARRAY

$A R A=1$ NUX $=12$ NUY $=44$ NUZ $=4$ FIIL 11R1 $5 \quad 43 Q 12 \quad 11 R 2 \quad 6 \quad 43 Q 12$

END FILL $\begin{array}{llllll}11 R 3 & 7 & 43 Q 12 & 11 R 4 & 8 & 43 Q 12\end{array}$

END ARRAY

READ PLOT

TIL $=' X-Y$ SLICE OF PNL4267C AT $Z=50^{\prime}$

$X U L=-45 . \quad Y U L=45.0 \quad Z U L=50$

$X L R=45 . \quad Y L R=-45 . \quad Z L R=50$ 
UAX $=1$ VDN $=-1 \quad \mathrm{NAX}=130 \quad \mathrm{NCH}==^{\prime} 1245786^{\prime}$ END

END PLOT

END DATA

END 
SCALE INPUT DECK FOR PNL-4267 EXPERIMENT 181

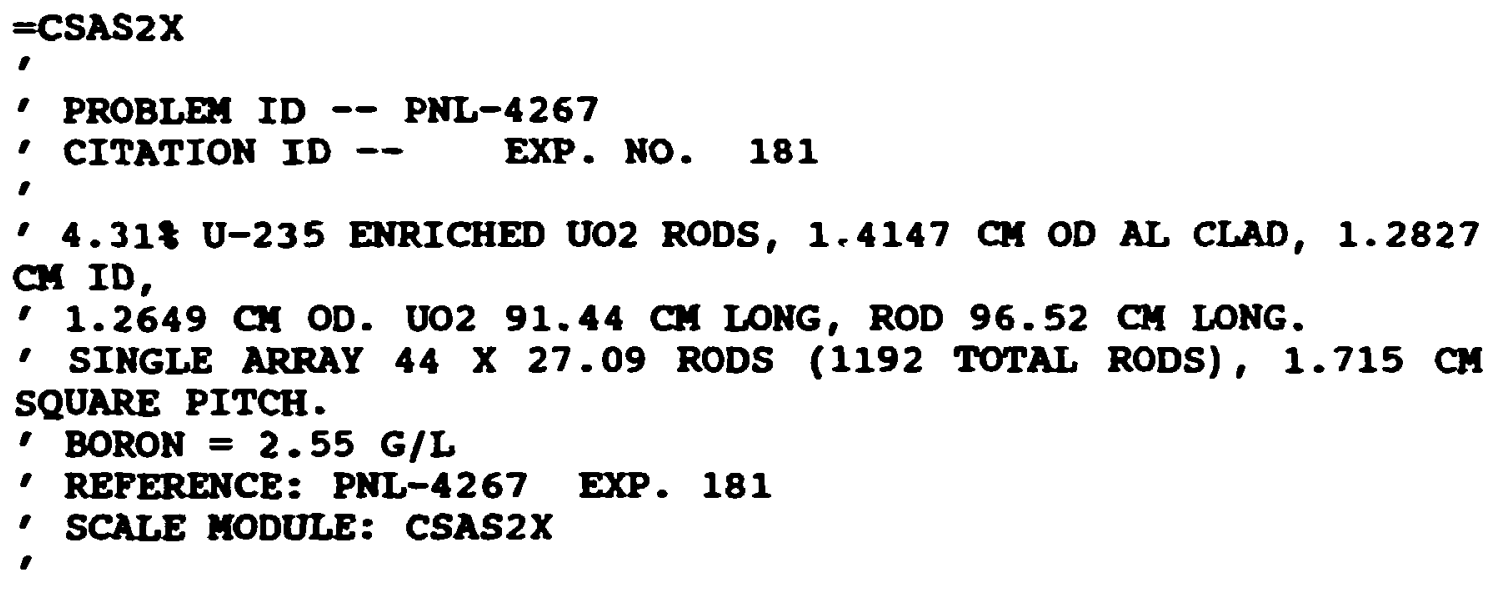

1.715 CH PITCH LATTICE CELL, ?.? WT: ENRICHED FUEL, ??000 $\mathrm{MHD} / \mathrm{T}$

27BURNUPLIB LATTICECELL

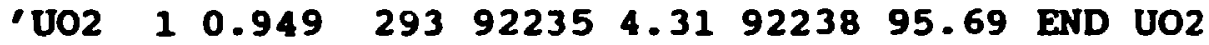

INSERT MIX 1 HERE

AL 21.0 END AI

H2O 31.0 END H2O

ARBIRUBBER $1.32160000601258 .1001 \quad 6.5 \quad 2000011.4$

160001.7801622 .1140000 .34 END

PLEXIGLASS 5 DEN $=.904$ END

H2O 6 END

BORON $7 \mathrm{DEN}=.002551 .0$ END

H2O 7 END

PLEXIGLASS 81.0 END

'UO2 $90.949 \quad 293922354.319223895 .69$ END UO2

INSERT MIX 9 HERE

AL 101.0 END AL

H2O 111.0 END H2O

BORON $11 \mathrm{DEN}=.002551 .0$ END

END COMP

- PITCH FOD F M COD C CID G

SQUAREPITCH $1.7151 .26499111 .4147 \quad 101.2827 \quad 0 \quad$ END

MORE DATA

RES $=1$ CYLINDER .63245 DAN $(1)=.269915$

END MORE

PNL-4267 EXP. $18144 \times 27.09$ ARRAY (1192 TOTAL RODS) BORON = $2.55 \mathrm{G} / \mathrm{L}$

READ PARY PLT $=Y E S$ RUN $=Y E S$ GEN $=205$ NPG $=600$ NSK=5 TME $=30$

WRS $=34$ RES $=205$ END PARM

READ GEOM

UNIT 1

'BOMTOM OF FUEL - RUBBER CAP IN LOWER LATTICE SUPPORT PLATE

CYLINDER 410.64135

CYLINDER 2 2 110.70735

CUBOID 514 PO.8575

$\begin{array}{ll}2.54 & 0.0 \\ 2.54 & 0.0 \\ 2.54 & 0.0\end{array}$


UNIT 2

- FUEL BETWEEN THE TWO LATTICE SUPPORT PLATES CYLINDER 1110.63245

CYLINDER 0110.64135

67.290 .0

67.290 .0

CYLINDER 210.70735

67.290 .0

CUBOID 71 1 4 P0.8575

67.290 .0

UNIT 3

' FUEL IN UPPER LATTICE SUPPORT PLATE

CYLINDER 110.63245

CYLINUER 0110.64135

CYLINDER 210.70735

CUBOID $\quad 5 \quad 14$ P0.8575

UNIT 4

- FUEL ABOVE THE UPPER LATTICE SUPPORT PLATE

CYLINDER 1110.63245

CYLINDER 0010.64135

CYLINDER 2110.70735

CUBOID 7 I 4 PP0.8575

$1.27 \quad 0.0$

$1.27 \quad 0.0$

$1.27 \quad 0.0$

$1.27 \quad 0.0$

UNIT 5

'BOTTOK OF SMEARED FUEL

$\begin{array}{llllllll}\text { CUBOID } 500 & 1 & 0.1559 & 0 & 2 P 0.8575 & 2.54 & 0.0\end{array}$

$\begin{array}{llllllll}\text { CUBOID } & 7 & 1 & 1.715 & 0 & 2 P 0.8575 & 2.54 & 0.0\end{array}$

UNIT 6

- SMEARED FUEL BETWEEN THE TWO LATTICE SUPPORT PLATES

$\begin{array}{lccllllll}\text { CUBOID } & 500 & 1 & 0.1559 & 0 & 2 P 0.8575 & 67.29 & 0.0 \\ \text { CUBOID } & 7 & 1 & 1.715 & 0 & 2 P 0.8575 & 67.29 & 0.0\end{array}$

UNIT 7

'SMEARED FUEL IN UPPER LATTICE SUPPORT PLATE

$\begin{array}{lllllllll}\text { CUBOID } & 500 & 1 & 0.1559 & 0 & 2 P 0.8575 & 1.27 & 0.0\end{array}$

$\begin{array}{llllllll}\text { CUBOID } & 7 & 1 & 1.715 & 0 & 2 \mathrm{PO} 0.8575 & 1.27 & 0.0\end{array}$

UNIT 8

'SMEARED FUEL ABOVE THE UPPER LATTICE SUPPORT PLATE

CUBOID $500 \quad 1 \quad 0.155900 \quad 2$ PO.8575

$\begin{array}{llllll}\text { CUBOID } \quad 7 & 1 & 1.715 & 0 & 2 P 0.8575\end{array}$

25.40 .0

GLOBAL

UNIT 9

ARRAY $1-24.01 \quad-37.73 \quad 0.0$

$\begin{array}{lllllll}\text { CUBOID } & 7 & 1 & 2 P 39.80 & 2 P 37.94 & 111.7 & 0.0\end{array}$

$\begin{array}{lllllll}\text { CUBOID } & 8 & 1 & 2 \text { P41.705 } & \text { 2P39.845 } & 111.7 & -17.105\end{array}$

REFLECTOR $6 \quad 2 \quad 4 R 3 \quad 2 R O \quad 10$

END GEOMETRY

READ BIAS ID $=500211$ END BIAS

READ ARRAY

$A R A=1$ NUX=28 NUY $=44 \quad N U Z=4 \quad$ FILL $27 R 1 \quad 5 \quad 43 Q 28 \quad 27 R 2 \quad 6 \quad 43028$

END FILL $\begin{array}{llllll}27 R 3 & 7 & 43 Q 28 & 27 R 4 & 8 & 43 Q\end{array}$

END ARRAY

READ PLOT

TTL $=' X-Y$ SLICE OF PNL4267D AT $Z=50^{\prime}$

$X U L=-45 . \quad Y U L=45.0 \quad Z U L=50$

$X L R=45 . \quad Y L R=-45 . \quad Z L R=50$ 
$\mathrm{UAX}=1 \mathrm{VDN}=-1$ NAX $=130 \mathrm{NCH}=\prime^{\prime} 124578 \&^{\prime}$ END

END PLOT

END DATA

END 
SCALE INPUT DECK FOR PNL-2438 EXPERIMENT 005

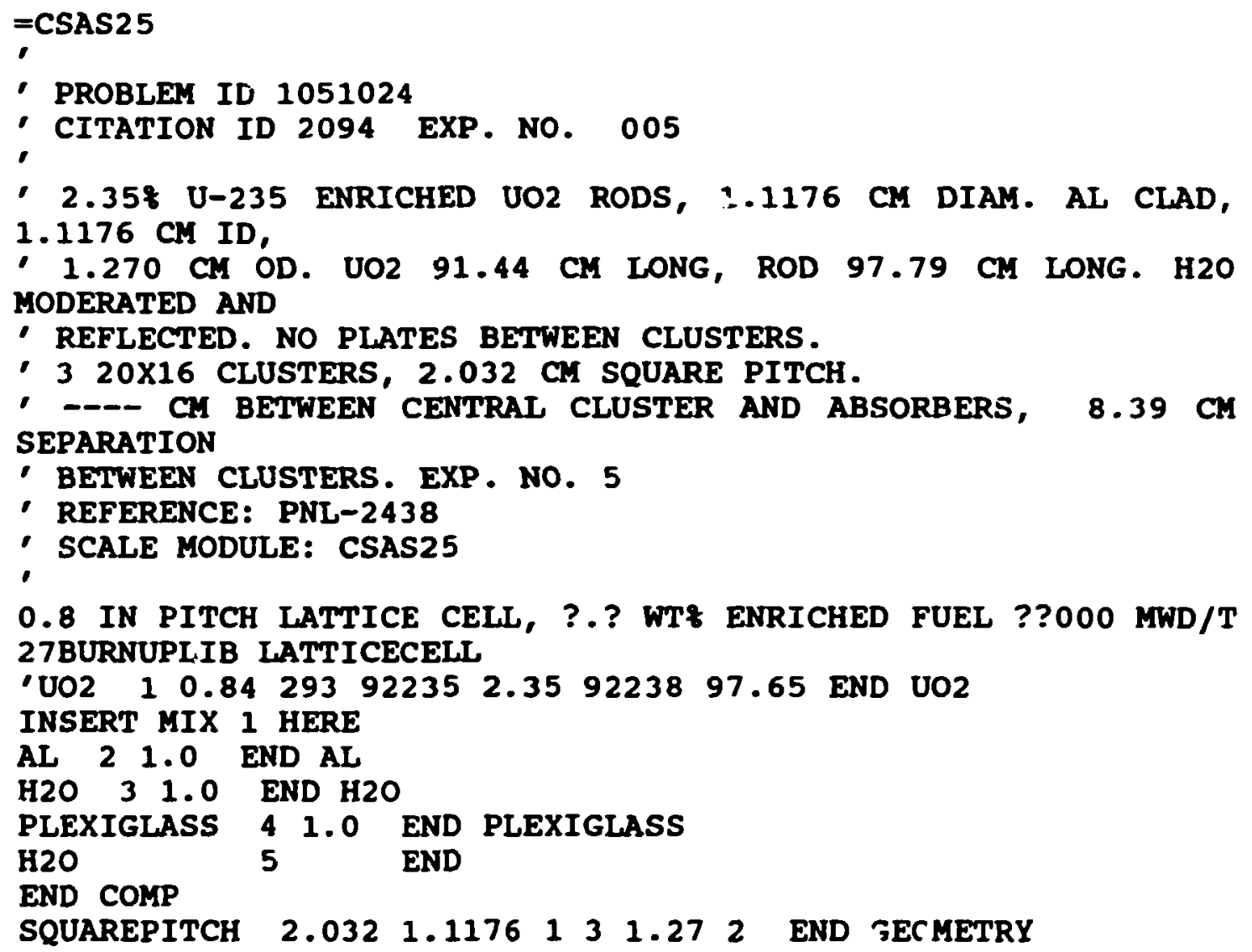

0.8 IN PITCH LATTICE CELL, ?.? WT' ENRICHED FUEL ??000 MWD/T 27BURNUPLIB LATTICECELL

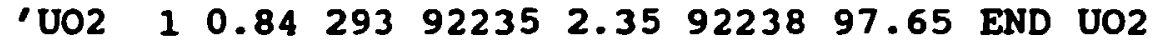

INSERT MIX 1 HERE

AL 21.0 END AL

$\mathrm{H} 2 \mathrm{O} 31.0$ END H2O

PLEXIGLASS 4 1.0 END PLEXIGLASS

H2O 5 END

END COMP

SQUAREPITCH $2.032 \quad 1.1176 \quad 1 \quad 3 \quad 1.27 \quad 2 \quad$ END FECMETRY

EXP\$5, 8.39 CM H2O SEPARATING 3 20X16 ARRAYS ?.? WT: ??000 MWD/T

READ PARM DLT=YES RUN=YES GEN=205 NPG=600 NSK=5 TME=25

WRS $=34$ RES $=205$ END PARM

READ GEOM

UNIT 1

CUBOID

UNIT 2

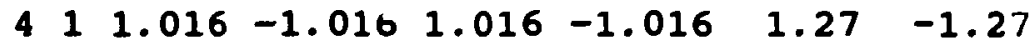

CYLINDER

91.440 .0

CYLINDER 210.635

$96.52-1.27$

CUBOID

UNIT 3

ARRAY $13 * 0.0$

UNIT 4

CUBOID

UNIT 5

ARRAY 2. $3 * 0.0$

UNIT 6

$\begin{array}{lllllllll}\text { CUBOID } & 5 & 1 & 8.39 & 0.0 & 32.512 & 0.0 & 97.79 & 0.0\end{array}$

GLOBAL UNIT 7

ARRAY $33 \star 0.0$

REFLECTOR $526 R 3.04 \quad 5$ 


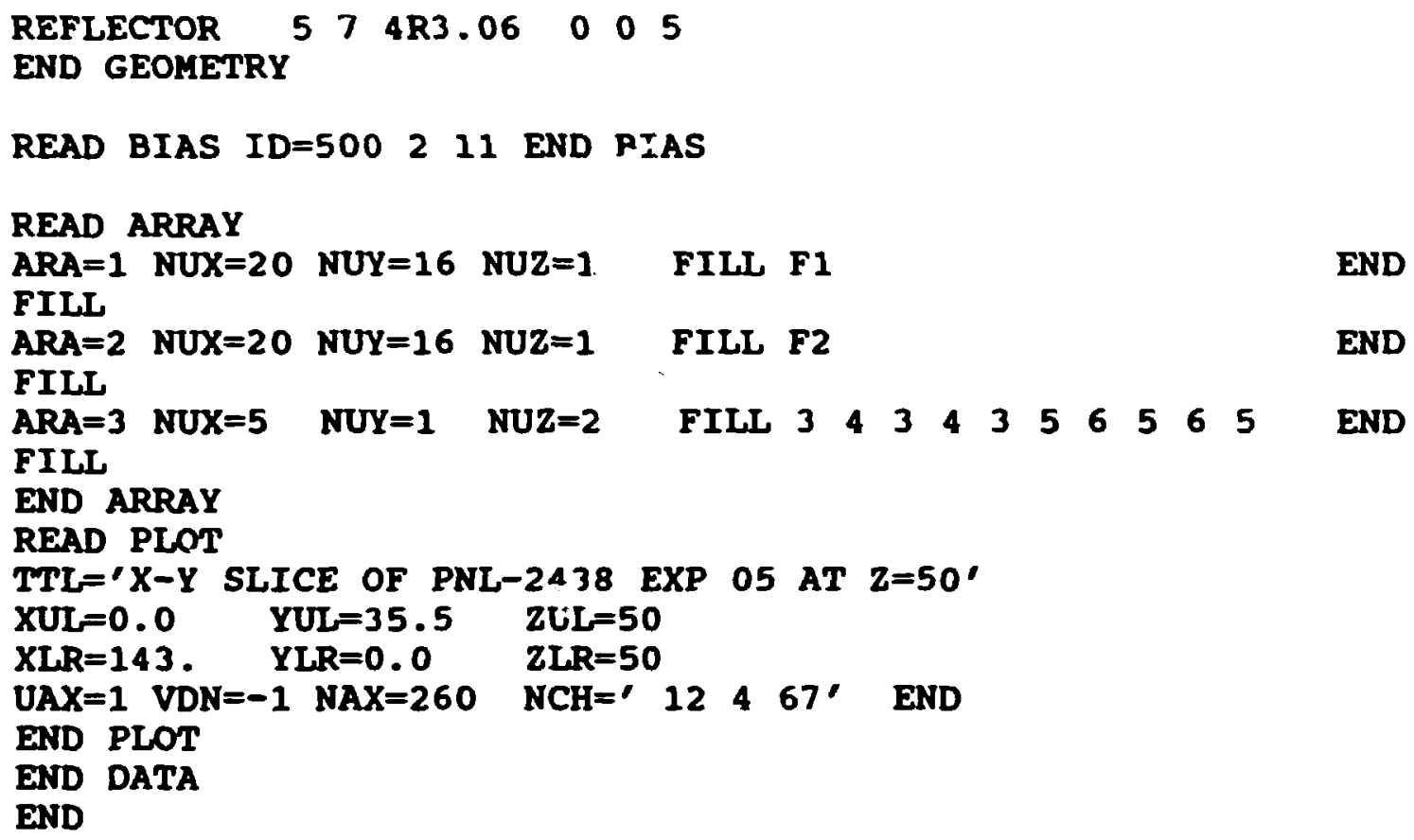


SCALE INPUT DECK FOR PNL-2438 EXPERIMENT 017

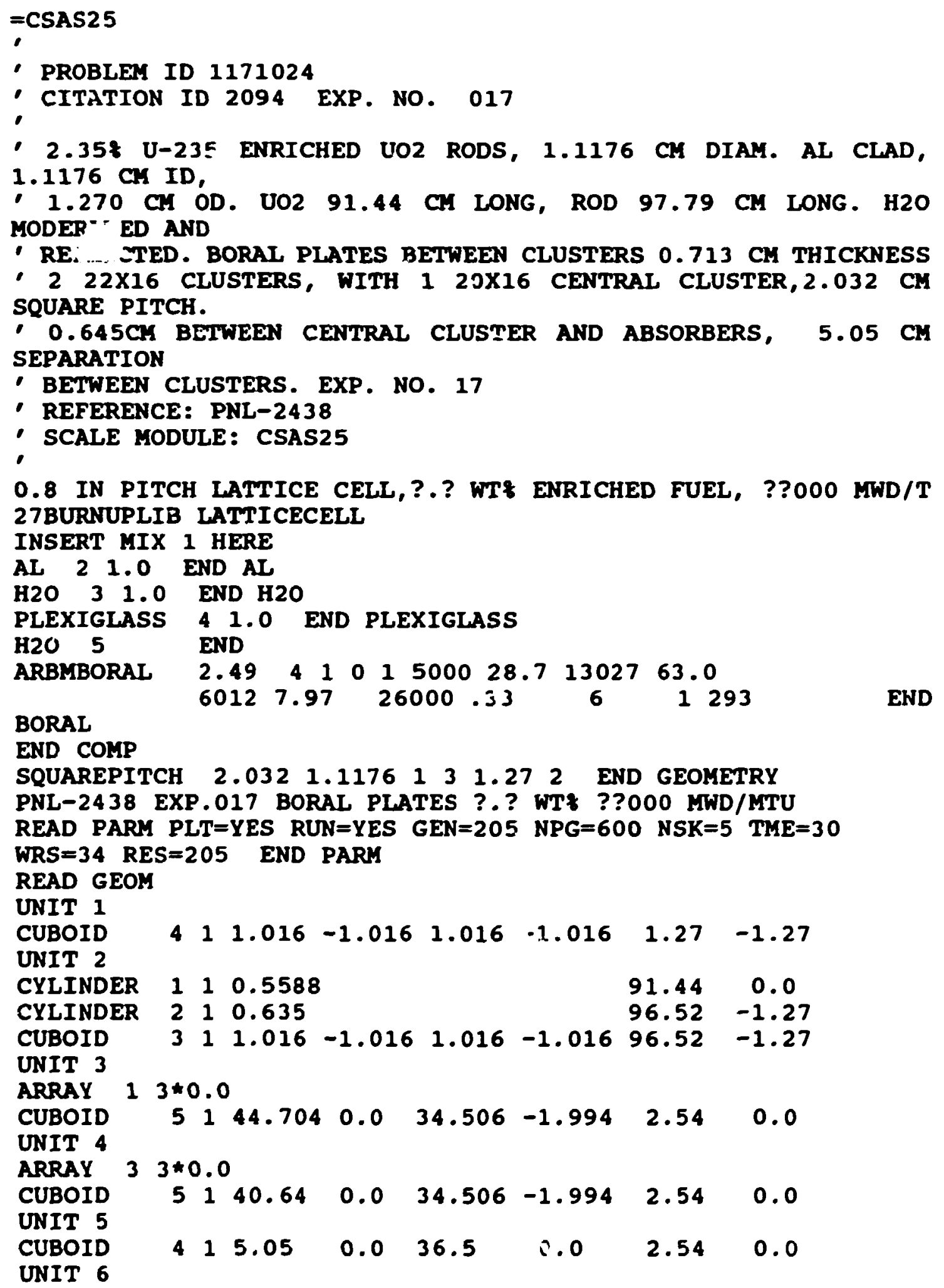




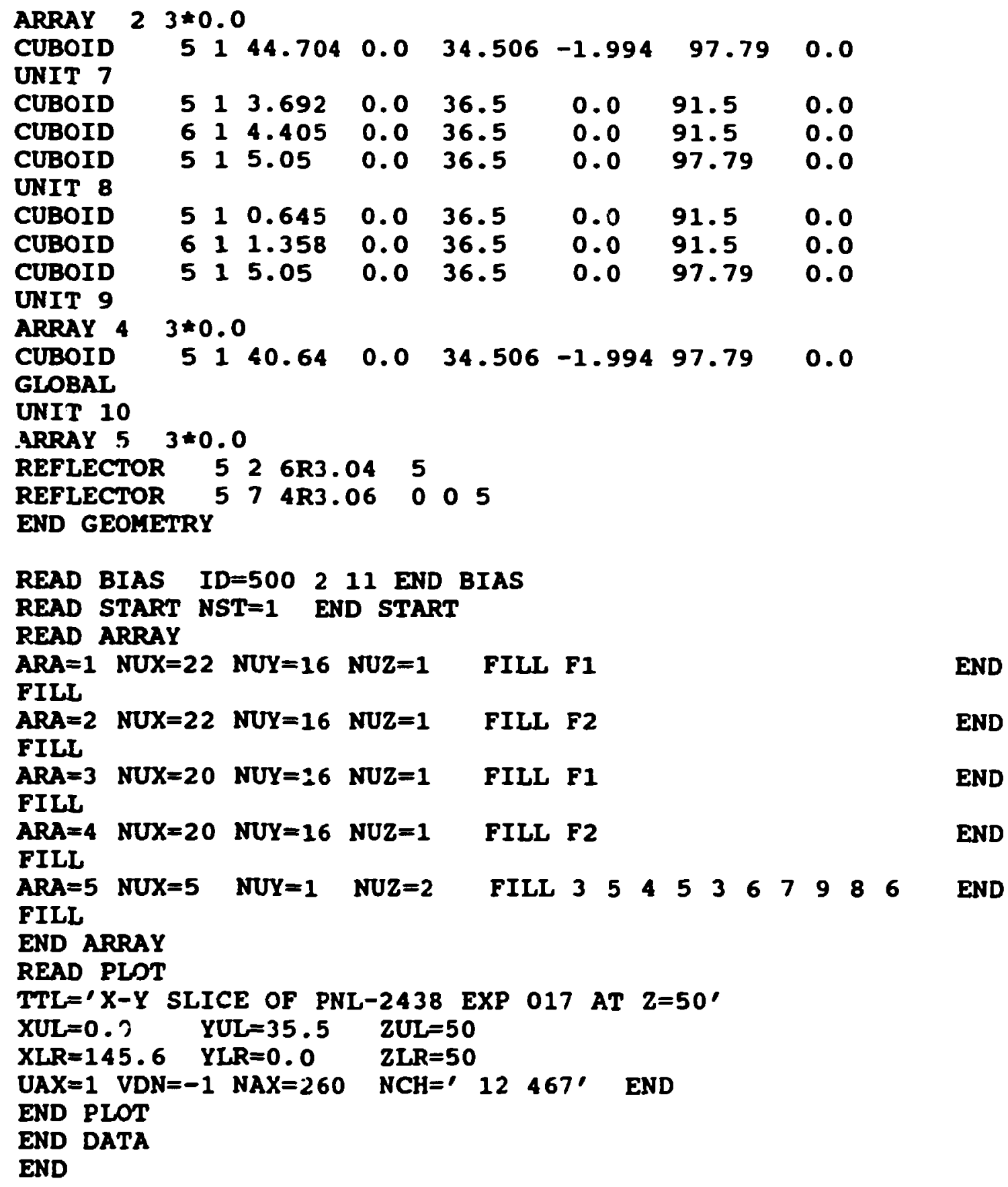


SCALE INPUT DECK FOR PNL-2438 EXPERIMENT 024

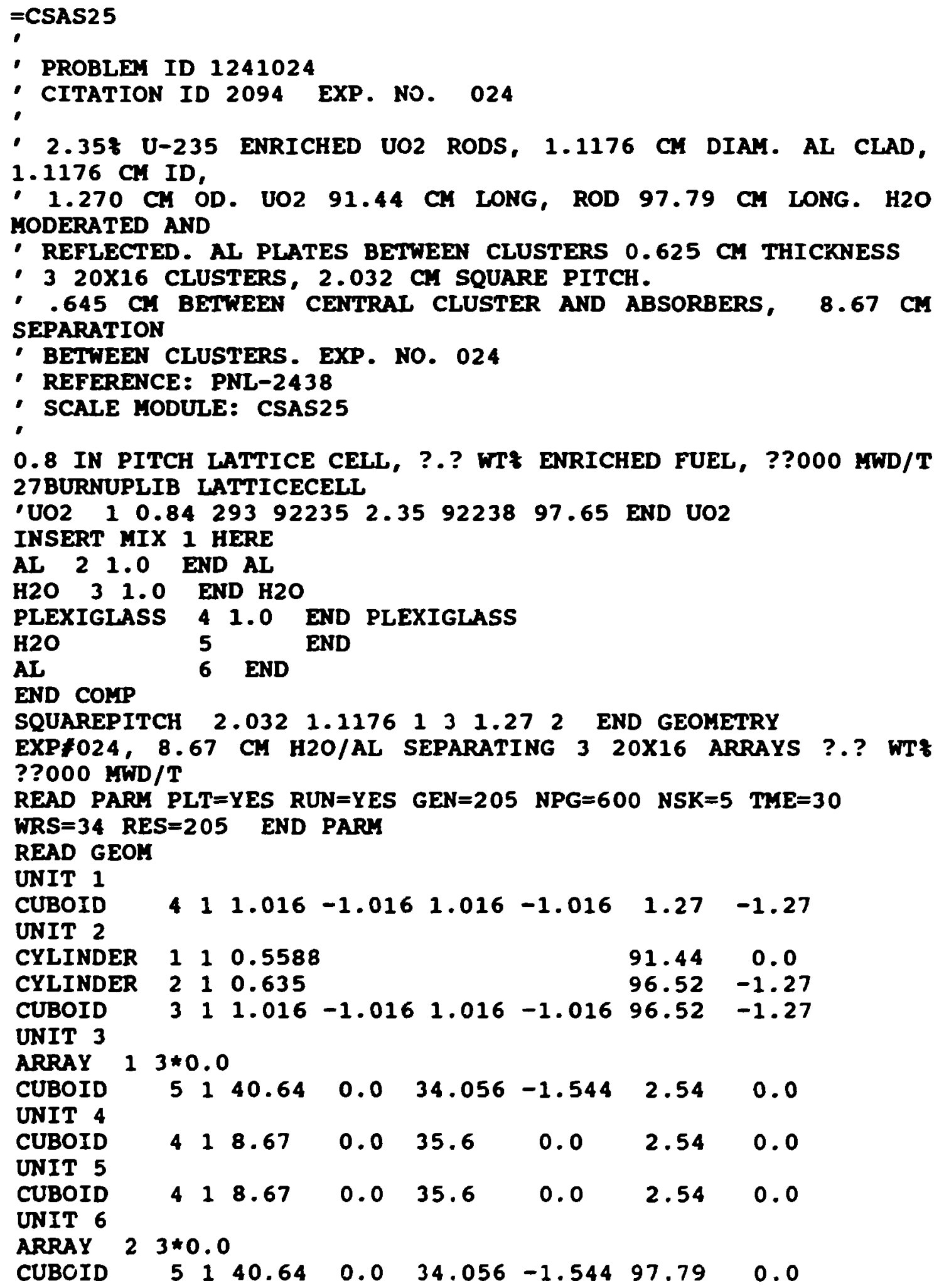




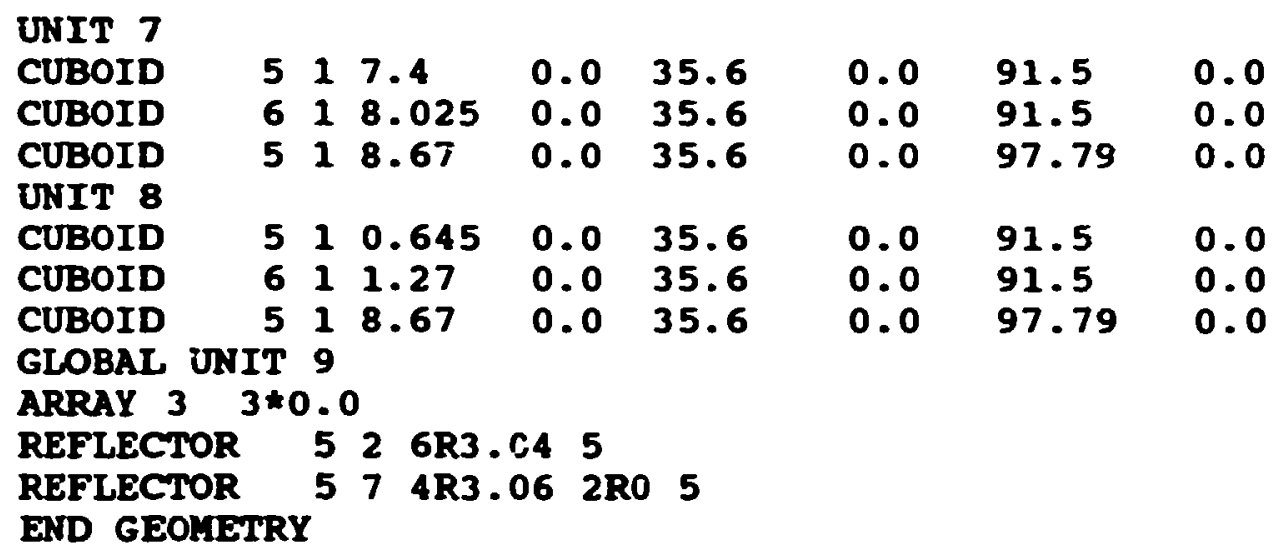


SCALE INPUT DECK FOR PNL-2438 EXPERIMENT 028

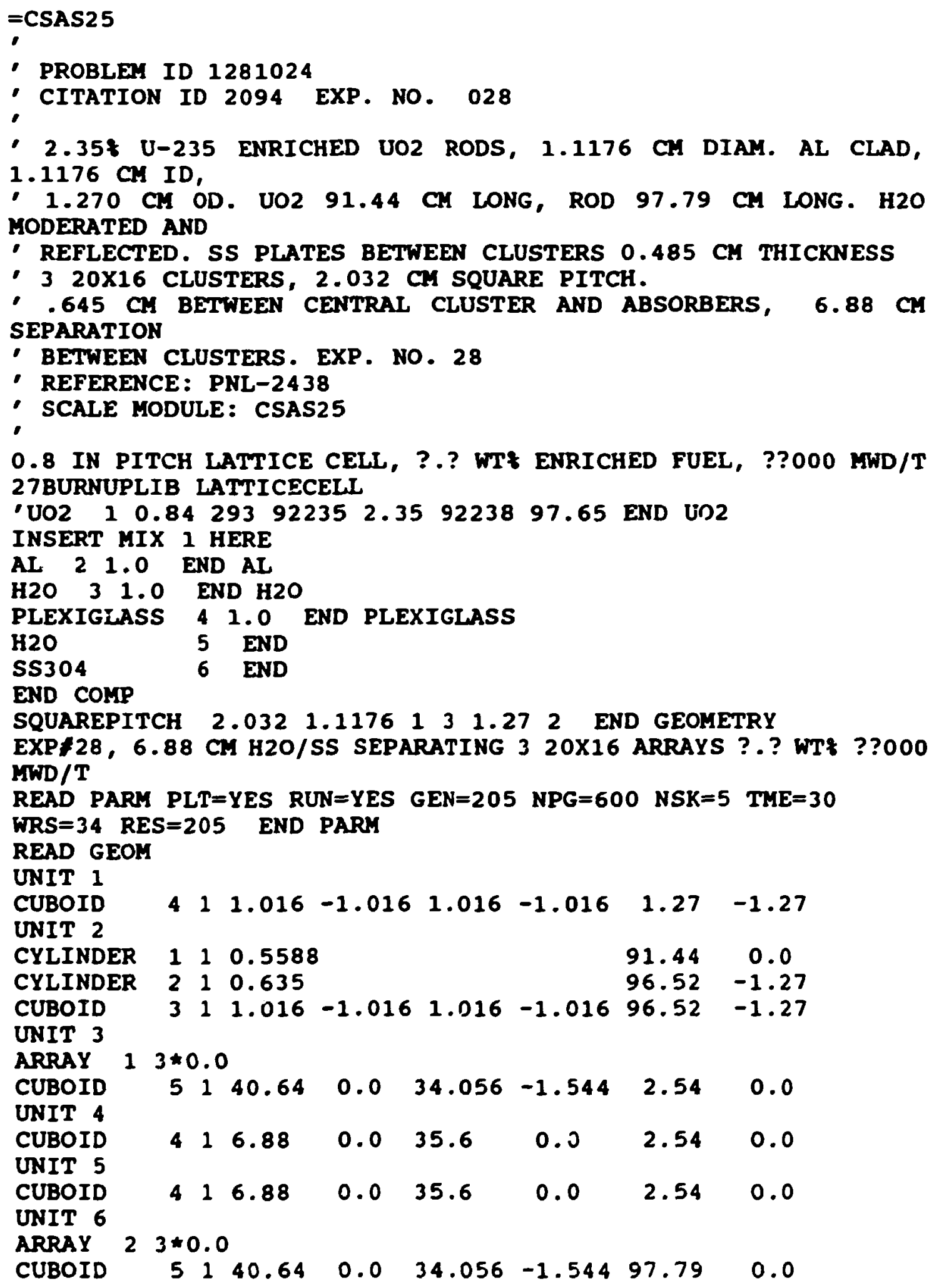




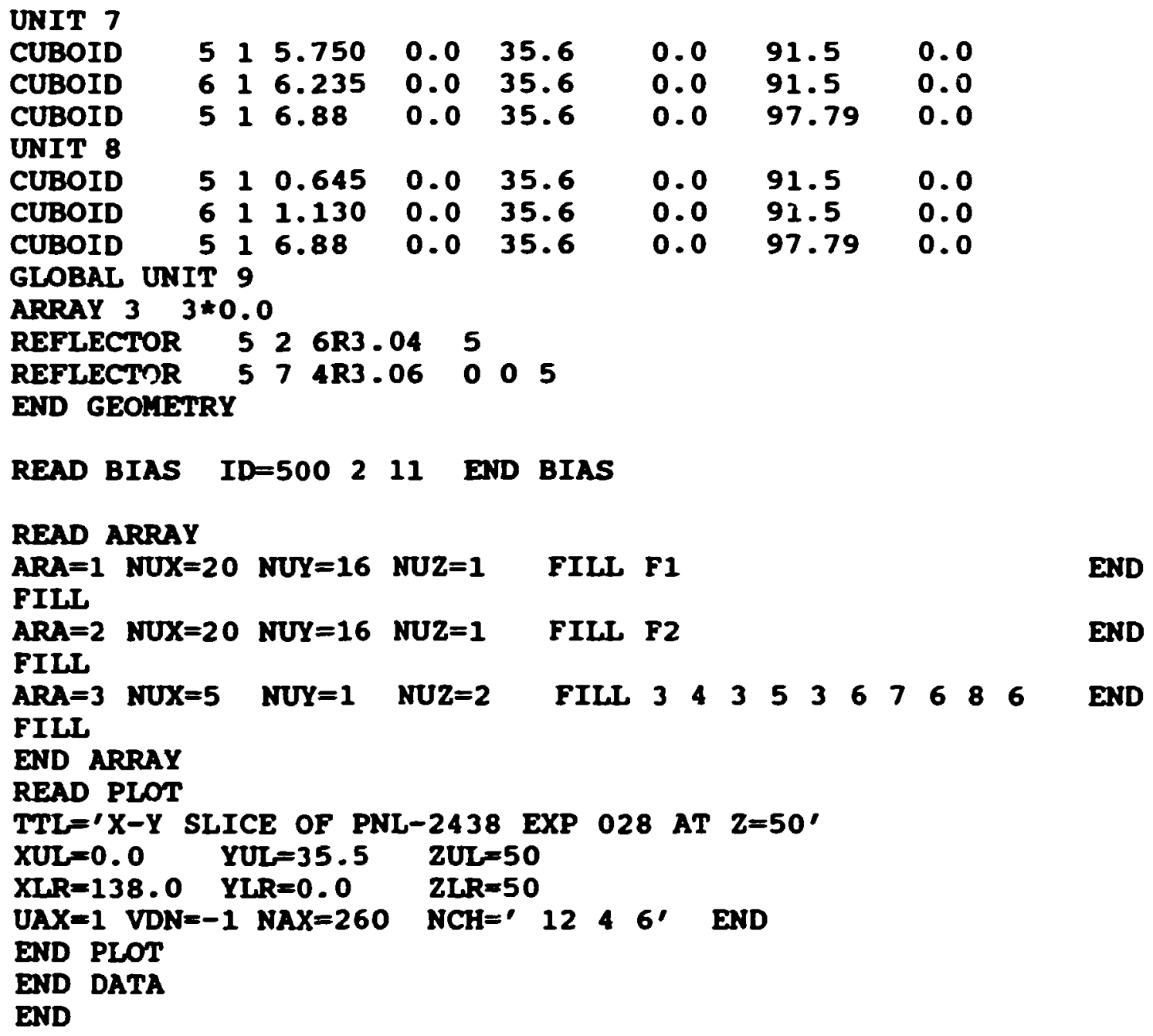


SCALE INPUT DECK FOR PNL-6838 EXPERIMENT TTC-5

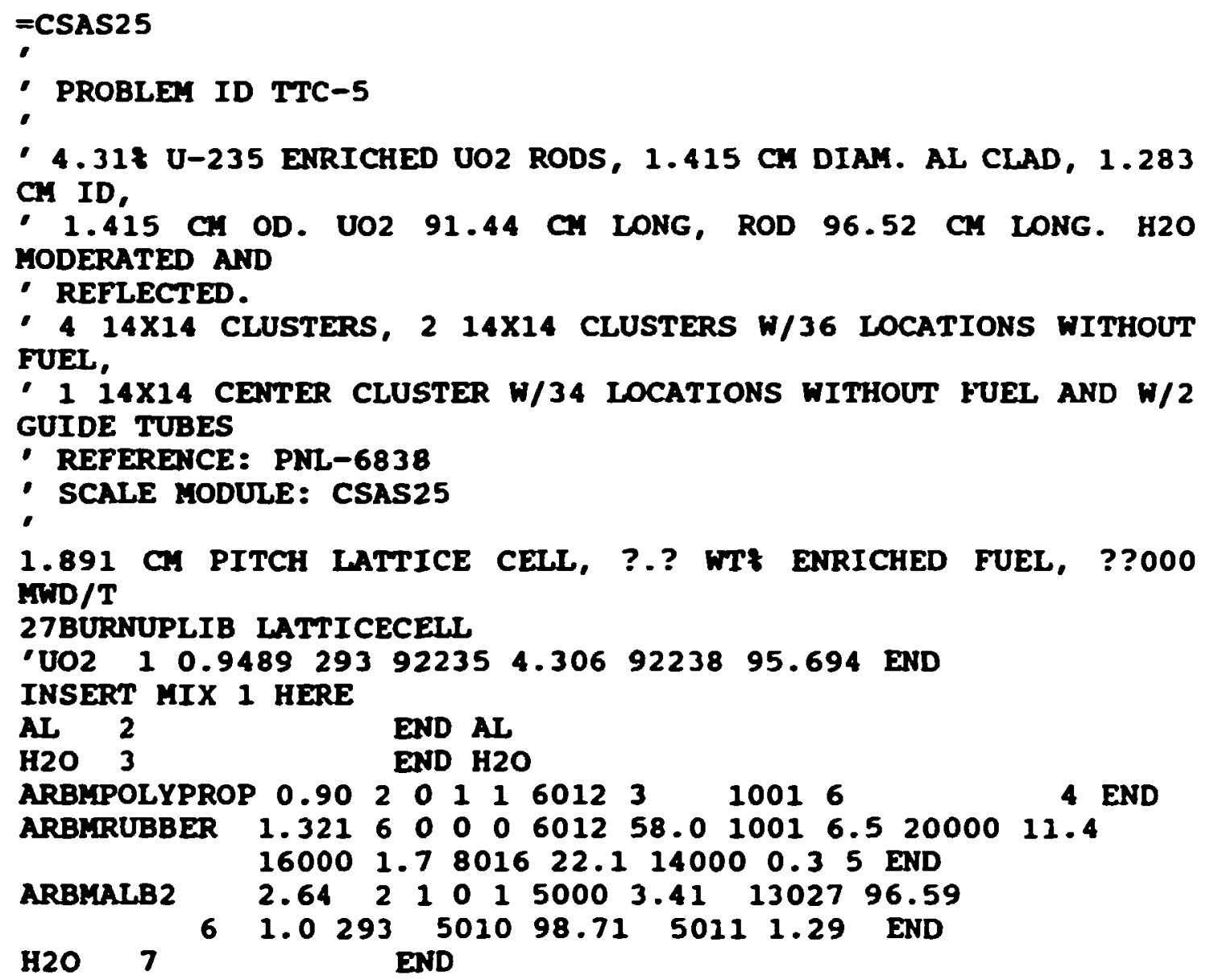

' 4.31 U-235 ENRICHED UO2 RODS, 1.415 CM DIAM. AL CLAD, 1.283 CM ID,

- 1.415 CH OD. UO2 91.44 Cr LONG, ROD 96.52 CH LONG. H2O MODERATED AND

- REFLECTED.

- $414 \times 14$ CLUSTERS, $214 \times 14$ CLUSTERS W/36 LOCATIONS WITHOUT FUEL,

- 1 14X14 CENTER CLUSTER W/34 LOCATIONS WITHOUT FUEL AND W/2 GUIDE TUBES

- REFERENCE: PNL-6838

- SCALE MODULE: CSAS25

-

1.891 CH PITCH LATTICE CELL, ?.? WT ENRICHED FUEL, ??000 MND/T

27BURNUPLIB LATTICECELL

'UO2 10.9489293922354 .3069223895 .694 END

INSERT MIX 1 HERE

AL

H2O 3

END AL

END H2O

$\begin{array}{llllllllllll}\text { ARBMPOLYPROP } & 0.90 & 2 & 0 & 1 & 1 & 6012 & 3 & 1001 & 6 & 4 & 4 \text { END }\end{array}$

ARBIRUBBER $1.321 \quad 6 \quad 0 \quad 0 \quad 0 \quad 6012 \quad 58.0 \quad 1001 \quad 6.5 \quad 20000 \quad 11.4$ $160001.7801622 .114000 \quad 0.35$ END

$\begin{array}{llllllllll}\text { ARBMALB2 } & 2.64 & 2 & 1 & 0 & 1 & 5000 & 3.41 & 13027 & 96.59\end{array}$

$\begin{array}{lllllllll}6 & 1.0293 & 5010 & 98.71 & 5011 & 1.29 & \text { END }\end{array}$

H2O 7 END

PLEXIGLASS 8 DEN $=1.185$ END

H2O 9 END

END COMP

SQUAREPITCH $\quad 1.891 \quad 1.265 \quad 1 \quad 3 \quad 1.415 \quad 2 \quad 1.283 \quad 0$ END

TTC-5 PNL-6838 1264 FUEL RODS ?.? WT ??000 MWD/T

READ PARM PLT=YES RUN=YES GEN=205 NPG=600 NSK=5 TME=45

WRS $=34$ RES $=205$ END PARY

READ GEOH

UNIT 1

$\begin{array}{lllllll}\text { CYLINDER } & 5 & 1 & .6415 & 1.23 & 0.0\end{array}$

$\begin{array}{llllll}\text { CYLINDER } & 2 & 1 & .7075 & 1.23 & 0.0\end{array}$

$\begin{array}{llllll}\text { CUBOID } 4 & 1 & 4 \mathrm{P} .9455 & 1.23 & 0.0\end{array}$

UNIT 2

$\begin{array}{llllll}\text { CYLINDER } & 1 & 1 & .6325 & 68.58 & 0.0\end{array}$

$\begin{array}{lllllll}\text { CYLINDER } & 0 & 1 & .6415 & 68.58 & 0.0\end{array}$

$\begin{array}{llllll}\text { CYLINDER } & 5 & 1 & .6415 & 68.58 & -1.31\end{array}$

$\begin{array}{lllllll}\text { CYLINDER } & 2 & 1 & .7075 & 68.58 & -1.31\end{array}$

$\begin{array}{llllll}\text { CUBOID } 31 & 4 P .9455 & 68.58 & -1.31\end{array}$

UNIT 3

$\begin{array}{llllll}\text { CYLINDER } & 1 & 1 & .6325 & 1.23 & 0.0\end{array}$ 


$\begin{array}{llllll}\text { CYLINDER } & 0 & 1 & .6415 & 1.23 & 0.0 \\ \text { CYLINDER } & 2 & 1 & .7075 & 1.23 & 0.0 \\ \text { CUBOID } & 4 & 1 & 4 P .9455 & 1.23 & 0.0 \\ \text { UNIT 4 } & & & & & \\ \text { CYLINDER } & 1 & 1 & .6325 & 21.63 & 0.0 \\ \text { CYLINDER } & 0 & 1 & .6415 & 21.63 & 0.0 \\ \text { CYLINDER } & 5 & 1 & .6415 & 24.17 & 0.0 \\ \text { CYLINDER } & 2 & 1 & .7075 & 24.17 & 0.0 \\ \text { CUBOID } & 3 & 1 & 4 P .9455 & 24.17 & 0.0 \\ & & & & & \\ \text { UNIT 5 } & & & & & \\ \text { CYLINDER } & 3 & 1 & .6415 & 1.23 & 0.0 \\ \text { CYLINDER } & 2 & 1 & .7075 & 1.23 & 0.0 \\ \text { CUBOID } & 4 & 1 & 4 P .9455 & 1.23 & 0.0 \\ \text { UNIT 6 } & & & & \\ \text { CYLINDER } & 3 & 1 & .6415 & 68.58 & -1.31 \\ \text { CYLINDER } & 2 & 1 & .7075 & 68.58 & -1.31 \\ \text { CUBOID } & 3 & 1 & 4 P .9455 & 68.58 & -1.31 \\ \text { UNIT 7 } & & & & & \\ \text { CYLINDER } & 3 & 1 & .6415 & 24.17 & 0.0 \\ \text { CYLINDER } & 2 & 1 & .7075 & 24.17 & 0.0 \\ \text { CUBOID } & 3 & 1 & 4 P .9455 & 24.17 & 0.0 \\ \text { UNIT 8 } & & & & & \\ \text { CYLINDER } & 3 & 1 & .7075 & 1.23 & 0.0 \\ \text { CUBOID } & 4 & 1 & 4 P .9455 & 1.23 & 0.0 \\ \text { UNIT 9 } & & & & & \\ \text { CUBOID } & 3 & 1 & 4 P .9455 & 68.58 & -1.31 \\ \text { UNIT 10 } & & & & & \\ \text { CUBOID } & 3 & 1 & 4 P .9455 & 24.17 & 0.0 \\ & & & & \end{array}$

UNIT 11

COM='FUEL ROD'

ARRAY $13 * 0.0$

UNIT 12

COY='GUIDE TUBE'

ARRAY $2 \quad 3 * 0.0$

UNIT 13

COY $=$ 'UNFILLED SPOT'

ARRAY $3 \quad 3 * 0.0$

UNIT 14

COY=' $14 \times 14$ ARRAY '

ARRAY 4 2*-13.237 0 .

CUBOID $31 \quad 4$ P13.2435

CUBOID 61 4P13.6275

96.520 .0

CUBOID $71 \quad 4$ P 16.1025

96.520 .0

96.520 .0

UNIT 15

COM=' $14 \times 14$ ARRAY WITH 36 MISSING'

ARRAY $52 \star-13.2370$.

CUBOID $31 \quad 4$ P13.2435

96.520 .0 


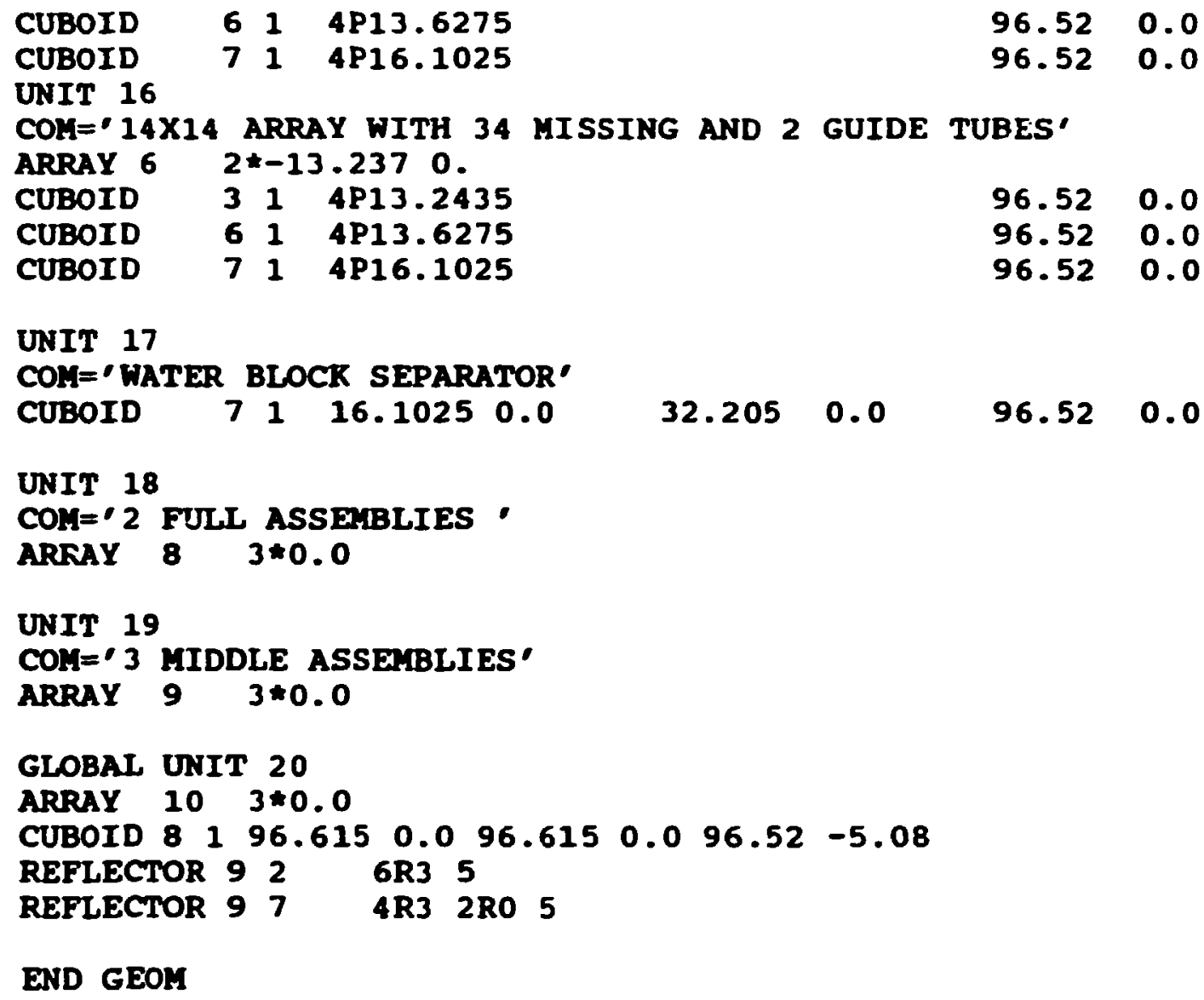




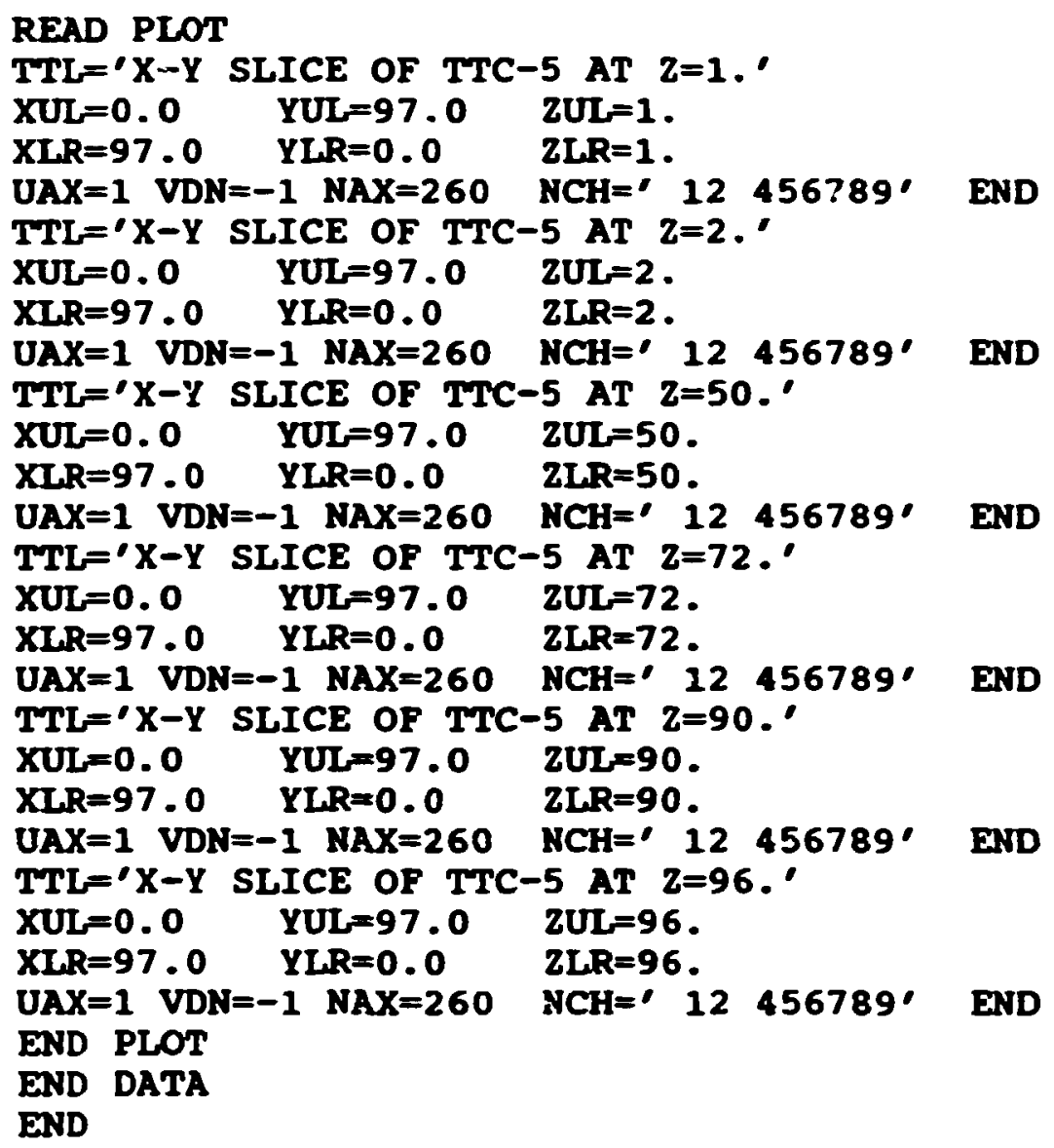

